\title{
Boundary trace of positive solutions of supercritical semilinear elliptic equations in dihedral domains
}

\author{
Moshe Marcus And LAURent VERON
}

\begin{abstract}
We study the generalized boundary value problem for (E) $-\Delta u+$ $|u|^{q-1} u=0$ in a dihedral domain $\Omega$, when $q>1$ is supercritical. The value of the critical exponent can take only a finite number of values depending on the geometry of $\Omega$. When $\mu$ is a bounded Borel measure in a $k$-wedge, we give necessary and sufficient conditions in order it be the boundary value of a solution of (E). We also give conditions which ensure that a boundary compact subset is removable. These conditions are expressed in terms of Bessel capacities $B_{S, q^{\prime}}$ in $\mathbb{R}^{N-k}$ where $s$ depends on the characteristics of the wedge. This allows us to describe the boundary trace of a positive solution of $(E)$.
\end{abstract}

Mathematics Subject Classification (2010): 35K60 (primary); 31A20, 31C15, 44A25, 46E35 (secondary).

\section{Introduction}

Let $\Omega$ be a bounded Lipschitz domain in $\mathbb{R}^{N}$ and let $q>1$. A long-term research on the equation

$$
-\Delta u+|u|^{q-1} u=0 \text { in } \Omega
$$

has been carried out for more than twenty years by probabilistic and/or analytic methods. Much of the research was focused on three main problems in domains of class $C^{2}$ :

(i) The Dirichlet problem for (1.1) with boundary data given by a finite Borel measure on $\partial \Omega$.

Both authors were partially sponsored by the French - Israeli cooperation program through grant No. 3-4299. The first author (MM) also wishes to acknowledge the support of the Israeli Science Foundation through grant No. 145-05.

Received October 18, 2013; accepted in revised form June 17, 2014.

Published online February 2016. 
(ii) The characterization of removable singular subsets of $\partial \Omega$ relative to positive solutions of (1.1).

(iii) The characterization of arbitrary positive solutions of (1.1) via an appropriate notion of boundary trace.

Consider the Dirichlet problem

$$
-\Delta u+|u|^{q-1} u=0 \quad \text { in } \Omega, u=\mu \text { in } \partial \Omega
$$

where $\mu \in \mathfrak{M}(\partial \Omega)$ (= space of finite Borel measures on $\partial \Omega$ ). Following [24], a (weak) solution $u:=u_{\mu}$ of (1.2) is a function $u \in L_{\rho}^{q}(\Omega)$ such that,

$$
\int_{\Omega}\left(-u \Delta \eta+\eta|u|^{q-1} u\right) d x=-\int_{\Omega} \mathbb{K}[\mu] \Delta \eta d x,
$$

for every in $\eta \in X(\Omega)$, where

$$
X(\Omega)=\left\{\eta: \rho^{-1} \Delta \eta \in L^{\infty}(\Omega)\right\} .
$$

Here $\mathbb{K}[\mu]$ is the harmonic function in $\Omega$ with boundary trace $\mu$ and $\rho$ is the first eigenfunction of $-\Delta$ in $W_{0}^{1,2}(\Omega)$ normalized so that $\max _{\Omega} \rho=1$. We also denote by $\lambda$ the corresponding eigenvalue. We recall that, if $\Omega$ is $\operatorname{Lipschitz} \mathbb{K}[\mu] \in L_{\rho}^{1}(\Omega)$; if $\Omega$ is of class $C^{2}, \mathbb{K}[\mu] \in L^{1}(\Omega)$.

A measure $\mu$ is a $q$-good measure if (1.2) has a solution. The space of $q$-good measures is denoted by $\mathfrak{M}_{q}(\partial \Omega)$. It is known that, if $\mu$ is $q$-good, the solution is unique. Furthermore, if $\mu$ satisfies the condition

$$
\int_{\Omega} \mathbb{K}[|\mu|]^{q} \rho d x<\infty
$$

then it is $q$-good. When $\mu$ satisfies this condition we say that it is a $q$-admissible measure.

When $\Omega$ is a domain of class $C^{2}, \mathbb{K}[\mu] \in L_{\rho}^{q}$ for every $q \in\left(1, \frac{N+1}{N-1}\right)$ and every $\mu \in \mathfrak{M}(\partial \Omega)$. Therefore, for $q$ in this range, every measure in $\mathfrak{M}(\partial \Omega)$ is $q$-good and there is no removable boundary set (except for the empty set). Problem (iii), for $q$ in this range, was resolved by Le Gall [16] (for $N=q=2$ ) and Marcus and Véron [19] (for $1<q<\frac{N+1}{N-1}, N \geq 3$ ).

The number $q_{c}=\frac{N+1}{N-1}$ is called the critical value for (1.1). If $q$ is supercritical, i.e. $q \geq q_{c}$, point singularities are removable. In particular there is no solution of (1.2) when $\mu=\delta_{y}$ (= a Dirac measure concentrated at a point $y \in \partial \Omega$ ).

In the supercritical case, problems (i)-(iii), $\Omega$ of class $C^{2}$, have been resolved in several stages. We say that a compact set $E \subset \partial \Omega$ is removable relative to equation (1.1) if there exists no positive solution vanishing on $\partial \Omega \backslash E$. We say that $E$ is conditionally removable if any solution $u$ of (1.2), with $\mu \in \mathfrak{M}(\partial \Omega)$, such that $u=0$ on $\partial \Omega \backslash E$ must vanish in $\Omega$. 
With respect to problem (ii) it was shown that a compact set $E \subset \partial \Omega$ is removable if and only if $C_{\frac{2}{q}, q^{\prime}}(E)=0, q^{\prime}=q /(q-1)$. Here $C_{\alpha, p}$ denotes the Bessel capacity, with the indicated indexes on $\partial \Omega$. (see Subsection 4.2 for an overview of Bessel capacities). This result was obtained by Le Gall [16] for $q=2$, Dynkin and Kuznetsov [8] for $1<q \leq 2$, Marcus and Véron [20] for $q>2$. For a unified analytic proof, covering all $q \geq q_{c}$ see [21].

The above result implies that every $q$-good measure $\mu$ must vanish on sets of $C_{\frac{2}{q}, q^{\prime}}$ capacity zero. On the other hand a result of Baras and Pierre [3] implies that every positive measure $\mu \in \mathfrak{M}(\partial \Omega)$ that vanishes on sets of $C_{\frac{2}{q}, q^{\prime}}$ capacity zero is the limit of an increasing sequence of admissible measures and therefore $q$-good. In conclusion: a measure $\mu \in \mathfrak{M}(\partial \Omega)$ is $q$-good if and only if it vanishes on sets of $C_{\frac{2}{q}, q^{\prime}}$ capacity zero. This takes care of problem (i).

Problem (iii) has been treated in several papers, with various definitions of a generalized boundary trace for positive solutions of (1.1), see [9] and [23]. Finally a full characterization of positive solutions was obtained by Mselati [25] for $q=2$, Dynkin [7] for $1<q<2$ and Marcus [18] for every $q \geq q_{c}$. In [7, 25] the restriction to $q \leq 2$ was dictated by their use of probabilistic techniques that do not apply to $q>2$. In [18] the proof is purely analytic.

If $\Omega$ is Lipschitz, $\xi \in \partial \Omega$, we say that $q_{\xi}$ is the critical value for (1.1) at $\xi$ if, for $1<q<q_{\xi}$, problem (1.2) with $\mu=\delta_{\xi}$ has a solution, but for $q>q \xi$ no such solution exists.

In contrast to the case of smooth domains, when $\Omega$ is Lipschitz, $q_{\xi}$ may vary with the point. For every compact set $F \subset \partial \Omega$ there exists a number $q(F)>1$ such that, for $1<q<q(F)$, every measure in $\mathfrak{M}(\partial \Omega)$ supported in $F$ is $q$-good. Obviously $q(F) \leq \min \left\{q_{\xi}: \xi \in F\right\}$ but it is not clear if equality holds.

In the special case when $\Omega$ is a polyhedron, the function $\xi \rightarrow q \xi$ obtains only a finite number of values (in fact, it is constant on each open face and each open edge) and, if $q \geq q_{\xi}$, an isolated singularity at $\xi$ is removable. Furthermore, the assumption $1<q<\min \left\{q_{\xi}: \xi \in \partial \Omega\right\}$ implies that every measure in $\mathfrak{M}(\partial \Omega)$ is $q$-good. For this and related results see [24].

In the present paper we study problem (1.2) when $\Omega$ is a polyhedron and $q$ is supercritical, i.e. $q \geq \min \left\{q_{\xi}: \xi \in \partial \Omega\right\}$. Following is a description of the main results.

\section{A. On the action of Poisson-type kernels with fractional dimension}

In preparation for the study of supercritical boundary value problem s we establish an harmonic analytic result, extending a well known result on the action of Poisson kernels on Besov spaces with negative index (see [28, 1.14.4.] and [4]). We first quote the classical result for comparison purposes.

Proposition 1.1. Let $1<q<\infty$ and $s>0$. Then, for any bounded Borel measure $\mu$ in $\mathbb{R}^{n-1}$,

$$
I(\mu)=\int_{\mathbb{R}_{+}^{n}}\left|\mathbb{K}_{n}[\mu](y)\right|^{q} e^{-y_{1}} y_{1}^{s q-1} d y \approx\|\mu\|_{B^{-s, q}\left(\mathbb{R}^{n-1}\right)}^{q} .
$$


Here $\mathbb{K}_{n}[\mu]$ denotes the Poisson potential of $\mu$ in $\mathbb{R}_{+}^{n}=\mathbb{R}_{+} \times \mathbb{R}^{n-1}$, namely,

$$
\mathbb{K}_{n}[\mu](y)=\gamma_{n} y_{1} \int_{\mathbb{R}^{n-1}} \frac{d \mu(z)}{\left(y_{1}^{2}+|\zeta-z|^{2}\right)^{n / 2}} \quad \forall y=\left(y_{1}, \zeta\right) \in \mathbb{R}_{+}^{n}
$$

where $\gamma_{n}$ is a constant depending only on $n$.

Notation. Let $m$ be a positive integer and let $v$ be a real number, $v \geq m+1$. Denote,

$$
\mathbb{K}_{v, m}[\mu](\tau, \zeta):=\int_{\mathbb{R}^{m}} \frac{\tau^{\nu-m} d \mu(z)}{\left(\tau^{2}+|\zeta-z|^{2}\right)^{\nu / 2}} \quad \forall \tau \in(0, \infty), \zeta \in \mathbb{R}^{m} .
$$

Note that

$$
\mathbb{K}_{n}[\mu]=\gamma_{n} \mathbb{K}_{n, n-1}[\mu] .
$$

Theorem 1.2. Let $m$ and $v$ be as above. Then, for every $q>1$ and every $s \in$ $\left(0, m / q^{\prime}\right), q^{\prime}=q /(q-1)$, there exists a positive constant $c$ such that, for every positive measure $\mu \in \mathfrak{M}\left(\mathbb{R}^{m}\right)$ supported in $B_{R / 2}(0)$ for some $R>1$,

$$
\begin{aligned}
\frac{1}{c}\|\mu\|_{B^{-s, q}\left(\mathbb{R}^{m}\right)}^{q} & \leq \int_{0}^{R}\left(\int_{|\zeta|<R}\left|\mathbb{K}_{\nu, m}[\mu](\tau, \zeta)\right|^{q} d \zeta\right) \tau^{s q-1} d \tau \\
& \leq c R^{(s+\nu-m) q+1}\|\mu\|_{B^{-s, q}\left(\mathbb{R}^{m}\right)}^{q} .
\end{aligned}
$$

This also holds when $s=m / q^{\prime}$, provided that the diameter of $\operatorname{supp} \mu$ is sufficiently small. tion.

This is proved in Section 3 (see Theorem 3.8) using a slightly different nota-

B. The critical value and the characterization of q-good measures in a k-wedge

The next step towards the study of boundary value problem $s$ in a polyhedron is the treatment of such problems in a $k$-wedge (or $k$-dihedron) i.e., the domain defined by the intersection of $k$ hyperplanes in $\mathbb{R}^{N}, 1<k<N$. The edge is an $(N-k)$ dimensional space.

We note that if $k=N$ the "edge" is a point and the corresponding wedge is a cone with vertex at this point. If $k=1$ the wedge is a half space. Both of these cases have been treated in [24].

Let $A$ be a Lipschitz domain in $S^{k-1}$. If

$$
S_{A}:=\left\{x \in \mathbb{R}^{N}:|x|=1, x \in A \times \prod_{j=k}^{N-1}[0, \pi]\right\} \subset S^{N-1}
$$

then

$$
D_{A}:=\left\{x=(r, \sigma): r>0, \sigma \in S_{A}\right\}
$$


is a $k$-wedge in $\mathbb{R}^{N}$ whose "edge" $d_{A}$ may be identified with $\mathbb{R}^{N-k}$ and its "opening" is $A$.

Let $\lambda_{A}$ be the first eigenvalue of $-\Delta_{S^{N-1}}$ in $W_{0}^{1,2}\left(S_{A}\right)$ and denote by $\kappa_{ \pm}$the roots of the equation,

$$
\kappa^{2}+(N-2) \kappa-\lambda_{A}=0 .
$$

Put

$$
q_{c}:=\frac{\kappa_{+}+N}{\kappa_{+}+N-2}
$$

and

$$
q_{c}^{*}:=1+\frac{2-k+\sqrt{(k-2)^{2}+4 \lambda_{A}-4(N-k) \kappa_{+}}}{\lambda_{A}-(N-k) \kappa_{+}} .
$$

Let $C_{\alpha, p}^{N-k}$ denote the Bessel capacity with the indicated indices in $\mathbb{R}^{N-k}$. The next theorem provides a characterization of $q$-good measures supported on $d_{A}$.

\section{Theorem 1.3.}

(a) If $1<q<q_{c}$ every measure in $\mathfrak{M}\left(d_{A}\right)$ is $q$-good relative to $D_{A}$. In fact every such measure is q-admissible.

(b) If $q \geq q_{c}^{*}$, the only $q$-good measure in $\mathfrak{M}\left(d_{A}\right)$ is the zero measure.

(c) If $q_{c} \leq q<q_{c}^{*}$, a measure $\mu \in \mathfrak{M}\left(d_{A}\right)$ is $q$-good relative to $D_{A}$ if and only if $\mu$ vanishes on every Borel set $E \subset d_{A} \operatorname{such}_{\text {that }} C_{s, q^{\prime}}^{N-k}(E)=0, s=2-\frac{k+\kappa_{+}}{q^{\prime}}$.

The characterization of $q$-good measures in a polyhedron follows as an easy consequence of the above theorem (see Theorem 4.6 below).

C. Characterization of removable sets

Let $\Omega$ be an $N$-dimensional polyhedron. Theorem 1.3 provides a necessary and sufficient condition for the removability of a singular set $E$ relative to the family of solutions $u$ such that

$$
\int_{\Omega}|u|^{q} \rho d x<\infty .
$$

The next result provides a necessary and sufficient condition for removability in the sense that the only non-negative solution $u \in C(\bar{\Omega} \backslash E)$ which vanishes on $\bar{\Omega} \backslash E$ is the trivial solution $u=0$.

Let $L$ denote a face or edge or vertex of $\Omega$ and put $k:=\operatorname{codim} L$. If $1<k<N$ let $d_{L}$ denote the linear space spanned by $L$, such that $L$ is an open subset of $d_{L}$. Let $Q_{L}$ denote the $k$-wedge with boundary $d_{L}$ such that, for some neighborhood $M$ of $L, \Omega \cap M=Q_{L} \cap M$ and let $A_{L}$ denote the opening of $Q_{L}$. If $k=N, Q_{L}$ is a cone with vertex $L$. Let $q_{c}(L)$ and $q_{c}^{*}(L)$ be defined as in (1.12) and (1.13) for $A=A_{L}$. Finally let

$$
s(L)=2-\frac{k+\kappa_{+}}{q^{\prime}}
$$

where $\kappa_{ \pm}$are the roots of (1.11) for $A=A_{L}$. If $k=N, Q_{L}$ is a cone with vertex $L$. In this case $q_{c}(L)=q_{c}^{*}(L)=1-\frac{2}{\kappa_{-}}$. If $k=1 q_{c}(L)=q_{c}^{*}(L)=(N+1) /(N-1)$. 
Theorem 1.4. Let $\Omega$ be a polyhedron in $\mathbb{R}^{N}$. A compact set $E \subset \partial \Omega$ is removable if and only if, for every $L$ as above such that $E \cap L \neq \emptyset$, the following conditions hold:

- if $1 \leq k<N$ : either $q_{c}(L) \leq q<q_{c}^{*}(L)$ and $C_{s(L), q^{\prime}}^{N-k}(E \cap L)=0$ or $q \geq q_{c}^{*}(L)$

- if $k=N: q \geq q_{c}(L)$.

The present paper is part of an article, "Boundary trace of positive solutions of semilinear elliptic equations in Lipschitz domains" arXiv:0907.1006 (2009). The first part of this article was published in [24]. The second and last part are presented here. The characterization of $q$-good measures, here established in polyhedrons, was recently established in [2], for arbitrary Lipschitz domains and a general family of nonlinearities. However the full removability result, Theorem 4.11, has not been superseded. (In [2] the authors provided - in the generality mentioned above - a characterization of conditional removability but not of full removability.) The methods of proof in the two papers are completely different. In the present paper, the characterization of $q$-good measures is based on an extension of a result of [4] and $[28,1.14 .4$.] on the action of Poisson kernels on Besov spaces with negative index. The use of Poisson-type kernels with fractional dimension has recently appeared in [12] to be a fundamental tool for the study of the boundary trace problem for semilinear elliptic equations with critical Hardy potentials depending on the distance to the boundary in the supercritical case. In [2] the proof relies on a relation between elliptic semilinear equations with absorption and linear Schrödinger equations.

\section{The Martin kernel and critical values in a $k$-dimensional dihedron.}

\subsection{The geometric framework}

An $N$-dim polyhedron $P$ is a bounded domain bordered by a finite number of hyperplanes. Thus the boundary of $P$ is the union of a finite number of sets $\left\{L_{k, j}: k=1, \ldots, N, j=1, \ldots, n_{k}\right\}$ where $\left\{L_{1, j}\right\}$ is the set of open faces of $P,\left\{L_{k, j}\right\}$ for $k=2, \ldots, N-1$, is the family of relatively open $N-k$-dimensional edges and $\left\{L_{N, j}\right\}$ is the family of vertices of $P$. An $N-k$-dimensional edge is a relatively open set in the intersection of $k$ hyperplanes; it will be described by the characteristic angles of these hyperplanes.

We recall that the spherical coordinates in $\mathbb{R}^{N}=\left\{x=\left(x_{1}, \ldots, x_{N}\right)\right\}$ are expressed by

$$
\left\{\begin{array}{l}
x_{1}=r \sin \theta_{N-1} \sin \theta_{N-2} \cdots \sin \theta_{2} \sin \theta_{1} \\
x_{2}=r \sin \theta_{N-1} \sin \theta_{N-2} \cdots \sin \theta_{2} \cos \theta_{1} \\
x_{3}=r \sin \theta_{N-1} \sin \theta_{N-2} \cdots \cos \theta_{2} \\
\vdots \\
x_{N-1}=r \sin \theta_{N-1} \cos \theta_{N-2}, \\
x_{N}=r \cos \theta_{N-1}
\end{array}\right.
$$


where $r=|x|, \theta_{1} \in[0,2 \pi]$ and $\theta_{\ell} \in[0, \pi]$ for $\ell=2,3, \ldots, N-1$. We denote $\sigma=\left(\theta_{1}, \ldots \theta_{N-1}\right)$. Thus in spherical coordinates $x=(r, \sigma)$.

We consider an unbounded non-degenerate $k$-dihedron, $2 \leq k \leq N$ defined as follows. Let $A$ be given by

$$
A= \begin{cases}\left(0, \alpha_{1}\right) \times \prod_{j=2}^{k-1}\left(\alpha_{j}, \alpha_{j}^{\prime}\right) & \text { if } k>2 \\ \left(0, \alpha_{1}\right) & \text { if } k=2\end{cases}
$$

where

$$
0<\alpha_{1}<2 \pi, \quad 0 \leq \alpha_{j}<\alpha_{j}^{\prime}<\pi \quad j=2, \ldots, k-1 .
$$

We denote by $S_{A}$ the spherical domain

$$
S_{A}=\left\{x \in \mathbb{R}^{N}:|x|=1, \sigma \in A \times \prod_{j=k}^{N-1}[0, \pi]\right\} \subset S^{N-1}
$$

and by $D_{A}$ the corresponding $k$-dihedron,

$$
D_{A}=\left\{x=(r, \sigma): r>0, \sigma \in S_{A}\right\} .
$$

The edge of $D_{A}$ is the $(N-k)$-dimensional space

$$
d_{A}=\left\{x: x_{1}=x_{2}=\ldots=x_{k}=0\right\} .
$$

\subsection{On the Martin kernel and critical values in a cone}

We recall here some elements of local analysis when $\Omega=C_{A} \cap B_{1}, A$ is a Lipschitz domain in $S^{N-1}$ and $C_{A}$ is the cone with vertex 0 and opening $A$.

Denote by $\lambda_{A}$ the first eigenvalue and by $\phi_{A}$ the first eigenfunction of $-\Delta^{\prime}$ in $W_{0}^{1,2}(A)$ (normalized by $\max \phi_{A}=1$ ). Let $\kappa_{-}$be the negative root of (1.11) and put

$$
\Phi_{1}(x):=\frac{1}{\gamma}|x|^{\kappa_{-}} \phi_{A}(x /|x|)
$$

where $\gamma$ is a positive number. Then $\Phi_{1}$ is a harmonic function in $C_{A}$ vanishing on $\partial C_{A} \backslash\{0\}$. We choose $\gamma=\gamma_{A}$ so that the boundary trace of $\Phi_{1}$ is $\delta_{0}$ (=Dirac measure on with mass 1 at the origin).

(i) If $q \geq 1-\frac{2}{\kappa_{-}}$, there is no solution of (1.1) in $\Omega_{S}$ with isolated singularity at 0 (see [10]).

(ii) If $1<q<1-\frac{2}{\kappa_{-}}$, then for any $k>0$ there exists a unique solution $u:=u_{k}$ to problem (1.2) with $\mu=k \delta_{0}$ and

$$
u_{k}(x)=k \Phi_{1}(x)(1+o(1)) \quad \text { as } x \rightarrow 0 .
$$

The function $u_{\infty}=\lim _{k \rightarrow \infty} u_{k}$ is a positive solution of (1.1) in $\Omega$ which vanishes on $\partial \Omega \backslash\{0\}$ and satisfies

$$
u_{\infty}(x)=|x|^{-\frac{2}{q-1}} \omega_{A}(x /|x|)(1+o(1)) \quad \text { as } x \rightarrow 0
$$


where $\omega_{A}$ is the (unique) positive solution of

$$
-\Delta^{\prime} \omega-a_{N, q} \omega+|\omega|^{q-1} \omega=0
$$

on $S^{N-1}$. Here $\Delta^{\prime}$ is the Laplace-Beltrami operator and

$$
a_{N, q}=\frac{2}{q-1}\left(\frac{2 q}{q-1}-N\right) .
$$

(iii) If $u \in C\left(\bar{\Omega}_{A} \backslash\{0\}\right)$ is a positive solution of (1.1) vanishing on $\left(\partial C_{A} \cap B_{r_{0}}(0)\right) \backslash$ $\{0\}$, then either $u$ satisfies (2.4) for some $k>0$ or $u$ satisfies (2.5). In particular there exists a unique positive solution vanishing on $\left(\partial C_{A} \cap B_{r_{0}}(0)\right) \backslash\{0\}$ with strong singularity at 0 . (For (ii) and (iii) see [24, Theorem 5.7].)

\subsection{Separable harmonic functions}

and the Martin kernel in a $k$-dihedron, $2 \leq k<N$

In the system of spherical coordinates, the Laplacian takes the form

$$
\Delta u=\partial_{r r} u+\frac{N-1}{r} \partial_{r} u+\frac{1}{r^{2}} \Delta_{S^{N-1}} u
$$

where the Laplace-Beltrami operator $\Delta_{S^{N-1}}$ is expressed by induction by

$$
\begin{aligned}
\Delta_{S^{N-1}} u= & \frac{1}{\left(\sin \theta_{N-1}\right)^{N-2}} \frac{\partial}{\partial \theta_{N-1}}\left(\left(\sin \theta_{N-1}\right)^{N-2} \frac{\partial u}{\partial \theta_{N-1}}\right) \\
& +\frac{1}{\left(\sin \theta_{N-1}\right)^{2}} \Delta_{S^{N-2}} u,
\end{aligned}
$$

and

$$
\Delta_{S^{1}} u=\partial_{\theta_{1} \theta_{1}} u .
$$

If we compute the positive harmonic functions in the $k$-dihedron $D_{A}$ of the form

$$
v(x)=v(r, \sigma)=r^{\kappa} \omega(\sigma) \quad \text { in } D_{A}, \quad v=0 \quad \text { in } \partial D_{A} \backslash\{0\},
$$

we find that $\omega$ must be a positive eigenfunction corresponding to the first eigenvalue, $\lambda_{A}$, of $-\Delta_{S^{N-1}}$ in $W_{0}^{1,2}\left(S_{A}\right)$,

$$
\begin{cases}\Delta_{S^{N-1}} \omega+\lambda_{A} \omega=0 & \text { in } S_{A} \\ \omega=0 & \text { on } \partial S_{A}\end{cases}
$$

and $\kappa$ must be a root of the algebraic equation (1.11) with $\lambda_{A}$ as above. Thus $\kappa=\kappa_{ \pm}$ where

$$
\begin{aligned}
& \kappa_{+}=\frac{1}{2}\left(2-N+\sqrt{(N-2)^{2}+4 \lambda_{A}}\right) \\
& \kappa_{-}=\frac{1}{2}\left(2-N-\sqrt{(N-2)^{2}+4 \lambda_{A}}\right) .
\end{aligned}
$$


Since

$$
S^{N-1}=\left\{\sigma=\left(\sigma_{2} \sin \theta_{N-1}, \cos \theta_{N-1}\right): \sigma_{2} \in S^{N-2}, \theta_{N-1} \in(0, \pi)\right\},
$$

we look for a solution $\omega=\omega^{\{1\}}$ of (2.10) of the form

$$
\omega^{\{1\}}(\sigma)=\left(\sin \theta_{N-1}\right)^{\kappa+} \omega^{\{2\}}\left(\sigma_{2}\right), \quad \theta_{N-1} \in(0, \pi), \quad \sigma_{2} \in S^{N-2} .
$$

Here $S^{N-2}=S^{N-1} \cap\left\{x_{N}=0\right\}$ and we denote

$$
S_{A}^{\{N-2\}}=S_{A} \cap\left\{x_{N}=0\right\}, \quad D_{A}^{\{N-2\}}:=D_{A} \cap\left\{x_{N}=0\right\} \subset \mathbb{R}^{N-1} .
$$

Then (2.11) jointly with relation (2.8) implies

$$
\begin{cases}\Delta_{S^{N-2}} \omega^{\{2\}}+\left(\lambda_{A}-\kappa_{+}\right) \omega^{\{2\}}=0 & \text { on } S_{A}^{\{N-2\}} \\ \omega^{\{2\}}=0 & \text { on } \partial S_{A}^{\{N-2\}} .\end{cases}
$$

Since we are interested in $\omega^{\{2\}}$ positive, $\lambda_{A}^{\{2\}}:=\lambda_{A}-\kappa_{+}$must be the first eigenvalue of $-\Delta_{S^{N-2}}$ in $W_{0}^{1,2}\left(S_{A}^{\{N-2\}}\right)$.

Next we look for positive harmonic functions $\tilde{u}$ in $D_{A}^{\{N-2\}}$ such that

$$
\tilde{u}\left(x_{1}, \ldots, x_{N-1}\right)=r^{\kappa^{\prime}} \omega\left(\sigma_{2}\right), \quad \tilde{u}=0 \text { on } \partial D_{A}^{\{N-2\}} .
$$

The algebraic equation which gives the exponents is

$$
\left(\kappa^{\prime}\right)^{2}+(N-3) \kappa^{\prime}-\lambda_{A}^{\{2\}}=0 .
$$

Denote by $\kappa_{+}^{\prime}$ the positive root of this equation. By the definition of $\lambda_{A}^{\{2\}}$,

$$
\kappa_{+}^{2}+(N-3) \kappa_{+}-\lambda_{A}^{\{2\}}=\kappa_{+}^{2}+(N-2) \kappa_{+}-\lambda_{A}=0 .
$$

Therefore $\kappa_{+}^{\prime}=\kappa_{+}$. Accordingly, if $k \geq 3$, we set

$$
\omega^{\{2\}}\left(\sigma_{2}\right)=\left(\sin \theta_{N-2}\right)^{\kappa_{+}} \omega^{\{3\}}\left(\sigma_{3}\right),
$$

and find that $\omega^{\{3\}}$ satisfies

$$
\begin{cases}\Delta_{S^{N-3}} \omega^{\{3\}}+\left(\lambda_{A}-2 \kappa_{+}\right) \omega^{\{3\}}=0 & \text { in } S_{A}^{\{N-3\}} \\ \omega^{\{3\}}=0 & \text { on } \partial S_{A}^{\{N-3\}},\end{cases}
$$

where

$$
S_{A}^{\{N-3\}}=S_{A} \cap\left\{x_{N}=x_{N-1}=0\right\} .
$$

Performing this reduction process $N-k$ times, we obtain the following results. 
(i) If $k>2$ then $\omega=\omega^{N-k}(\sigma)$ is given by

$$
\omega(\sigma)=\left(\sin \theta_{N-1} \sin \theta_{N-2} \ldots \sin \theta_{k}\right)^{\kappa+} \omega^{\{N-k+1\}}\left(\sigma_{N-k+1}\right)
$$

where

$$
\sigma_{N-k+1} \in S^{k-1}=S^{N-1} \cap\left\{x_{N}=, x_{N-1}=\cdots=x_{k+1}=0\right\}
$$

and $\omega^{\prime}:=\omega^{\{N-k+1\}}$ satisfies

$$
\begin{cases}\Delta_{S^{k-1}} \omega^{\prime}+\left(\lambda_{A}-(N-k) \kappa_{+}\right) \omega^{\prime}=0, & \text { in } S_{A}^{\{k-1\}} \\ \omega^{\prime}=0, & \text { on } \partial S_{A}^{\{k-1\}},\end{cases}
$$

where $S_{A}^{\{k-1\}}=S_{A} \cap\left\{x_{N}=x_{N-1}=\ldots=x_{k+1}=0\right\} \approx A$ and $\lambda_{A}-(N-k) \kappa_{+}$ is the first eigenvalue of the problem.

(ii) If $k=2$ then

$$
\omega(\sigma)=\left(\sin \theta_{N-1} \sin \theta_{N-2} \ldots \sin \theta_{2}\right)^{\kappa_{+}} \omega^{\{N-1\}}\left(\theta_{1}\right)
$$

where $\sigma_{N-1} \in S^{1} \approx \theta_{1} \in(0,2 \pi)$, and $\omega^{\{N-1\}}$ satisfies

$$
\begin{cases}\Delta_{S^{1}} \omega^{\{N-1\}}+\left(\lambda_{A}-(N-2) \kappa_{+}\right) \omega^{\{N-1\}}=0 & \text { on } S_{A}^{\{1\}} \\ \omega^{\{N-1\}}=0 & \text { on } \partial S_{A}^{\{1\}},\end{cases}
$$

with $\partial S_{A}^{\{1\}} \approx(0, \alpha)$. In this case

$$
\kappa_{+}=\frac{\pi}{\alpha}, \quad \omega^{\{N-1\}}\left(\theta_{1}\right)=\sin \left(\pi \theta_{1} / \alpha\right),
$$

and, by (1.11),

$$
\lambda_{A}-(N-2) \kappa_{+}=\frac{\pi^{2}}{\alpha^{2}} \Longrightarrow \lambda_{A}=\frac{\pi^{2}}{\alpha^{2}}+(N-2) \frac{\pi}{\alpha} .
$$

Observe that $\frac{1}{2} \leq \kappa_{+}$with equality holding only in the degenerate case $\alpha=2 \pi$ (which we exclude).

In either case, we find a positive harmonic function $v_{A}$ in $D_{A}$, vanishing on $\partial D_{A}$, of the form

$$
v_{A}(x)=|x|^{\kappa_{+}} \omega(x /|x|)
$$

with $\omega$ as in (2.14) (for $k>2$ ) or (2.18) (for $k=2$ ). Furthermore, if $\Omega$ is a domain in $\mathbb{R}^{N}$ such that, for some $R>0, \Omega \cap B_{R}(0)=D_{A} \cap B_{R}(0)$ and $w$ is a positive harmonic function in $\Omega$ vanishing on $d_{A} \cap B_{R}(0)$ then $w \sim v_{A}$ in $\Omega \cap B_{R^{\prime}}(0)$ for every $R^{\prime} \in(0, R)$. 
Similarly we find a positive harmonic function in $D_{A}$ vanishing on $\partial D_{A} \backslash\{0\}$, singular at the origin, of the form

$$
K_{A}^{\prime}(x)=|x|^{\kappa_{-}} \omega(x /|x|)
$$

If $\Omega$ is a domain as above and $z$ is a positive harmonic function in $\Omega$ vanishing on $d_{A} \cap B_{R}(0) \backslash\{0\}$ then $z \sim K_{A}^{\prime}$ in $\Omega \cap B_{R^{\prime}}(0) \backslash\{0\}$ for every $R^{\prime} \in(0, R)$.

As $K_{A}^{\prime}$ is a kernel function of $-\Delta$ at 0 it follows that $K_{A}^{\prime}$ is, up to a multiplicative constant $c_{A}$, the Martin kernel of $-\Delta$ in $D_{A}$, with singularity at 0 . The Martin kernel, with singularity at a point $z \in d_{A}$, is given by

$$
K_{A}(x, z)=c_{A} \frac{\left(\sin \theta_{N-1} \sin \theta_{N-2} \ldots \sin \theta_{k}\right)^{\kappa+} \omega^{\{N-k+1\}}\left(\sigma_{N-k+1}\right)}{|x-z|^{N-2+\kappa_{+}}}
$$

for every $x \in D_{A}$. From (2.1)

$$
\sin \theta_{N-1} \sin \theta_{N-2} \ldots \sin \theta_{k}=|x-z|^{-1} \sqrt{x_{1}^{2}+x_{2}^{2}+\ldots+x_{k}^{2}} .
$$

Therefore, if we write $x \in \mathbb{R}^{N}$ in the form $x=\left(x^{\prime}, x^{\prime \prime}\right), x^{\prime}=\left(x_{1}, \ldots, x_{k}\right), x^{\prime \prime}=$ $\left(x_{k+1}, \cdots, x_{N}\right)$, we obtain the formula,

$$
\begin{aligned}
K_{A}(x, z) & =c_{A} \frac{\left|x^{\prime}\right|^{\kappa_{+}} \omega^{\{N-k+1\}}\left(\sigma_{N-k+1}\right)}{|x-z|^{\left(N-2+2 \kappa_{+}\right)}} \\
& =c_{A} \frac{\left|x^{\prime}\right|^{\kappa_{+}} \omega^{\{N-k+1\}}\left(\sigma_{N-k+1}\right)}{\left(\left|x^{\prime}\right|^{2}+\left|x^{\prime \prime}-z\right|^{2}\right)^{\left(N-2+2 \kappa_{+}\right) / 2}} .
\end{aligned}
$$

Therefore, the Poisson potential of a measure $\mu \in \mathfrak{M}\left(d_{A}\right)$ is expressed by

$$
\begin{aligned}
\mathbb{K}_{A}[\mu](x)= & c_{A}\left|x^{\prime}\right|^{\kappa_{+}} \omega^{\{N-k+1\}}\left(\sigma_{N-k+1}\right) \\
& \times \int_{\mathbb{R}^{N-k}} \frac{d \mu(z)}{\left(\left|x^{\prime}\right|^{2}+\left|x^{\prime \prime}-z\right|^{2}\right)^{\left(N-2+2 \kappa_{+}\right) / 2}} .
\end{aligned}
$$

\subsection{The admissibility condition}

Consider the boundary value problem

$$
\left\{\begin{array}{l}
-\Delta u+|u|^{q-1} u=0 \quad \text { in } D_{A} \\
u=\mu \in \mathfrak{M}\left(\partial D_{A}\right)
\end{array}\right.
$$

Let

$$
\Gamma_{R}=\left\{x=\left(x^{\prime}, x^{\prime \prime}\right):\left|x^{\prime}\right| \leq R,\left|x^{\prime \prime}\right| \leq R\right\}, \quad D_{A, R}:=D_{A} \cap \Gamma_{R}
$$


and let $\rho_{R, A}$ denote the first (positive) eigenfunction in $D_{A, R}:=D_{A} \cap \Gamma_{R}$. In the rest of this section we drop the index $A$ in $K_{A}, \rho_{A, R}$ etc., except for $D_{A}, D_{A, R}$ and $d_{A}$.

First we observe that a positive Radon measure on $d_{A}$ is $q$-good relative to $D_{A}$ if and only if, for every compact set $F \subset d_{A}, \mu \chi_{F}$ is $q$-good in $D_{A}$

Now suppose that $\mu$ is compactly supported in $d_{A}$ and denote its support by $F$. We claim that $\mu$ is $q$-good in $D_{A}$ if and only if it is $q$-good relative to $D_{A, R}$ for all sufficiently large $R$. Let $R$ be such that $F \subset B_{R / 2}^{N-k}(0)$. Assume that $\mu$ is $q$-good in $D_{A, R}$. Let $v_{R}$ be the solution of (1.1) in $D_{A, R}$ such that $v_{R}=\mu$ on $d_{A} \cap \Gamma_{R}$, $v_{R}=0$ on $\partial D_{A, R} \backslash d_{A}$. Then $v_{R}$ increases with $R$ and $v=\lim _{R \rightarrow \infty} v_{R}$ is a solution of (1.1) in $D_{A}$ with boundary data $\mu$. This proves our claim in one direction; the other direction is obvious.

The condition for $\mu$ to be q-admissible in $D_{A, R}$ is

$$
\int_{D_{A, R}} \mathbb{K}^{R}[|\mu|](x)^{q} \rho_{R}(x) d x<\infty
$$

where $K^{R}$ is the Martin kernel of $-\Delta$ in $D_{A, R}$. If $R$ is sufficiently large then, in a neighborhood of $F, K^{R} \sim K$ and $\rho^{R} \sim \rho \sim v_{A}$. Therefore, a sufficient condition for $\mu$ to be $q-\operatorname{good}$ in $D_{A}$ is

$$
\left.\int_{\Gamma_{R} \cap D_{A}} \mathbb{K}[|\mu|](x)\right|^{q} \rho(x) d x<\infty \quad \forall R>0 .
$$

By the first observation in this subsection, it follows that the previous statement remains valid for any positive Radon measure supported on $d_{A}$.

By (2.21),

$$
\mathbb{K}[|\mu|](x) \leq c_{A}\left(r^{\prime}\right)^{\kappa+} \int_{\mathbb{R}^{N-k}} j\left(x^{\prime}, x^{\prime \prime}-z\right) d|\mu|(z)
$$

where

$$
j(x)=|x|^{-N+2-2 \kappa_{+}} \quad \forall x \in \mathbb{R}^{N} .
$$

Therefore, using (2.20), condition (2.27) becomes

$$
\int_{0}^{R} \int_{\left|x^{\prime \prime}\right|<R}\left(\int_{\mathbb{R}^{N-k}} j\left(x^{\prime}, x^{\prime \prime}-z\right) d|\mu|(z)\right)^{q}\left(r^{\prime}\right)^{(q+1) \kappa_{+}+k-1} d x^{\prime \prime} d r^{\prime}<\infty
$$

for every $R>0$.

\subsection{The critical values}

Relative to the equation

$$
-\Delta u+|u|^{q-1} u=0
$$

there exist two thresholds of criticality associated with the edge $d_{A}$. 
The first is the value $q_{c}^{*}$ such that, for $q_{c}^{*} \leq q$ the whole edge $d_{A}$ is removable but for $1<q<q_{c}^{*}$ there exist non-trivial solutions in $D_{A}$ which vanish on $\partial D_{A} \backslash d_{A}$. The second $q_{c}<q_{c}^{*}$ corresponds to the removability of points on $d_{A}$. For $q \geq q_{c}$ points on $d_{A}$ are removable while for $1<q<q_{c}$ there exist solutions with isolated point singularities on $d_{A}$. In the next two propositions we determine these critical values.

Proposition 2.1. Assume $q>1,1 \leq k<N$. Then the condition

$$
q<q_{c}^{*}:=1+\frac{2-k+\sqrt{(k-2)^{2}+4 \lambda_{A}-4(N-k) \kappa_{+}}}{\lambda_{A}-(N-k) \kappa_{+}}
$$

is necessary and sufficient for the existence of a non-trivial solution $u$ of (2.31) in $D_{A}$ which vanishes on $\partial D_{A} \backslash d_{A}$. Furthermore, when this condition holds, there exist non-trivial positive bounded measures $\mu$ on $d_{A}$ such that $\mathbb{K}[\mu] \in L_{\rho}^{q}\left(\Gamma_{R} \cap D_{A}\right)$.

Remark. The statement remains true for $k=N$, which is the case of the cone. In this case $q_{c}=q_{c}^{*}=1-\left(2 / \kappa_{-}\right)$and a straightforward computation yields:

$$
q_{c}=\frac{N+2+\sqrt{(N-2)^{2}+4 \lambda_{A}}}{N-2+\sqrt{(N-2)^{2}+4 \lambda_{A}}} .
$$

Proof. Recall that $\lambda_{A}-(N-k) \kappa_{+}$is the first eigenvalue in $S_{A}^{\{k-1\}}$ (see (2.15) and the remarks following it). Let $\kappa_{+}^{\prime}, \kappa_{-}^{\prime}$ be the two roots of the equation

$$
X^{2}+(k-2) X-\left(\lambda_{A}-(N-k) \kappa_{+}\right)=0,
$$

i.e.,

$$
\kappa_{ \pm}^{\prime}=\frac{1}{2}\left(2-k \pm \sqrt{(k-2)^{2}+4\left(\lambda_{A}-(N-k) \kappa_{+}\right.}\right) .
$$

Then, by [24, Theorem 5.7], recalled in Subsection 2.2, if $1<q<1-\left(2 / \kappa_{-}^{\prime}\right)$ there exists a unique solution of (2.31) in the cone $C_{S_{A}^{k-1}}$ i.e. the cone with opening $S_{A}^{k-1} \subset S^{k-1} \subset \mathbb{R}^{k}$ with trace $a \delta_{0}$ (where $\delta_{0}$ denotes the Dirac measure at the vertex of the cone and $a>0)$. By (2.5) this solution satisfies

$$
u_{a}(x)=a|x|^{-\alpha} \phi(x /|x|)(1+o(1)) \quad \text { as } x \rightarrow 0,
$$

where $\phi$ is the first positive eigenfunction of $-\Delta^{\prime}$ in $W_{0}^{1,2}\left(S_{A}^{k-1}\right)$ normalized so that $u_{1}$ possesses trace $\delta_{0}$.

The function $u$ given by

$$
\tilde{u}_{a}\left(x^{\prime}, x^{\prime \prime}\right)=u_{a}\left(x^{\prime}\right) \quad \forall\left(x^{\prime}, x^{\prime \prime}\right) \in D_{A}=C_{S_{A}^{k-1}} \times \mathbb{R}^{N-k},
$$

is a nonzero solution of (2.31) in $D_{A}$ which vanishes on $\partial D_{A} \backslash d_{A}$ and has bounded trace on $d_{A}$.

A simple calculation shows that $1-\left(2 / \kappa_{-}^{\prime}\right)$ equals $q_{c}^{*}$ as given in $(2.32)$. 
Next, assume that $q \geq q_{c}^{*}$ and let $u$ be a solution of (2.31) in $D_{A}$ which vanishes on $\partial D_{A} \backslash d_{A}$.

Given $\epsilon>0$ let $v_{\epsilon}$ be the solution of (2.31) in $D_{A}^{\{N-k-1\}} \backslash\left\{x^{\prime} \in \mathbb{R}^{k}:\left|x^{\prime}\right| \leq \epsilon\right\}$ such that

$$
v_{\epsilon}\left(x^{\prime}\right)= \begin{cases}0, & \text { if } x^{\prime} \in \partial D_{A}^{\{N-k-1\}},\left|x^{\prime}\right|>\epsilon \\ \infty, & \text { if }\left|x^{\prime}\right|=\epsilon\end{cases}
$$

Given $R>0$ let $w_{R}$ be the maximal solution in $\left\{x^{\prime \prime} \in \mathbb{R}^{N-k}:\left|x^{\prime \prime}\right|<R\right\}$.

Then the function $u^{*}$ given by

$$
u^{*}\left(x^{\prime}, x^{\prime \prime}\right)=v_{\epsilon}\left(x^{\prime}\right)+w_{R}\left(x^{\prime \prime}\right)
$$

is a supersolution of (2.31) in $D_{A} \backslash\left\{\left(x^{\prime}, x^{\prime \prime}\right):\left|x^{\prime}\right|>\epsilon,\left|x^{\prime \prime}\right|<R\right\}$ and it dominates $u$ in this domain. But $w_{R}\left(x^{\prime \prime}\right) \rightarrow 0$ as $R \rightarrow \infty$ and, by [10], $v_{\epsilon}\left(x^{\prime}\right) \rightarrow 0$ as $\epsilon \rightarrow 0$. Therefore $u_{+}=0$ and, by the same token, $u_{-}=0$.

Proposition 2.2. Let $A$ be defined as before. Then

$$
\mathbb{K}[\mu] \in L_{\rho}^{q}\left(\Gamma_{R} \cap D_{A}\right) \quad \forall \mu \in \mathfrak{M}\left(d_{A}\right), \quad \forall R>0
$$

if and only if

$$
1<q<q_{c}:=\frac{\kappa_{+}+N}{\kappa_{+}+N-2} .
$$

This statement is equivalent to the following:

Condition (2.36) is necessary and sufficient in order that the Dirac measure $\mu=\delta_{P}$, supported at a point $P \in d_{A}$, satisfy (2.35).

Proof. It is sufficient to prove the result relative to the family of measures $\mu$ such that $\mu$ is positive, has compact support and $\mu\left(d_{A}\right)=1$. Let $R>1$ be sufficiently large so that the support of $\mu$ is contained in $\Gamma_{R / 2}$. The measure $\mu$ can be approximated (in the sense of weak convergence of measures) by a sequence $\left\{\mu_{n}\right\}$ of convex combinations of Dirac measures supported in $d_{A} \cap \Gamma_{R / 2}$. For such a sequence $\mathbb{K}\left[\mu_{n}\right] \rightarrow \mathbb{K}[\mu]$ pointwise and $\left\{\mathbb{K}\left[\mu_{n}\right]\right\}$ is uniformly bounded in $D_{A} \backslash \Gamma_{3 R / 4}$. Therefore it is sufficient to prove the result when $\mu=\delta_{0}$. In this case the admissibility condition (1.5)) is

$$
\int_{0}^{R} \int_{\left|x^{\prime \prime}\right|<R} j(x)^{q}\left(r^{\prime}\right)^{(q+1) \kappa_{+}+k-1} d x^{\prime \prime} d r^{\prime}<\infty
$$

i.e.,

$$
\int_{0}^{R} \int_{0}^{R}|x|^{q\left(2-N-2 \kappa_{+}\right)}\left(r^{\prime}\right)^{(q+1) \kappa_{+}+k-1}\left(r^{\prime \prime}\right)^{N-k-1} d r^{\prime \prime} d r^{\prime}<\infty
$$


Substituting $\tau:=r^{\prime \prime} / r^{\prime}$ the condition becomes

$$
\int_{0}^{R} \int_{0}^{R / r^{\prime}}\left(1+\tau^{2}\right)^{\frac{q}{2}\left(2-N-2 \kappa_{+}\right)}\left(r^{\prime}\right)^{q\left(2-N-\kappa_{+}\right)+\kappa_{+}+N-1} \tau^{N-k-1} d \tau d r^{\prime}<\infty .
$$

This holds if and only if $q<\left(\kappa_{+}+N\right) /\left(\kappa_{+}+N-2\right)$.

Remark. It is interesting to notice that $k$ does not appear explicitly in (2.36). Furthermore, we observe that

$$
\frac{2}{q_{c}-1}\left(\frac{2 q_{c}}{q_{c}-1}-N\right)=\lambda_{A} \Longleftrightarrow \kappa_{+}\left(\kappa_{+}+N-2\right)=\lambda_{A},
$$

which follows from (2.11). This implies that there does not exist a nontrivial solution of the nonlinear eigenvalue problem

$$
\begin{aligned}
-\Delta_{S^{N}-1} \psi-\frac{2}{q-1}\left(\frac{2 q}{q-1}-N\right) \psi+|\psi|^{q-1} \psi=0 & \text { in } S_{D_{A}} \\
\psi=0 & \text { in } \partial S_{D_{A}}
\end{aligned}
$$

which, in turn, implies that there does not exists a nontrivial solution of (2.31) of the form $u(x)=u(r, \sigma)=|x|^{-2 /(q-1)} \psi(\sigma)$, and also no solution of this equation in $D_{A}$ which vanishes on $\partial D_{A} \backslash\{0\}$. This is the classical ansatz for the removability of isolated singularities in $d_{A}$.

\section{The harmonic lifting of a Besov space $B^{-s, p}\left(d_{A}\right)$}

Denote by $W^{\sigma, p}\left(\mathbb{R}^{\ell}\right)(\sigma>0,1 \leq p \leq \infty)$ the Sobolev spaces over $\mathbb{R}^{\ell}$. In order to use interpolation, it is useful to introduce the Besov space $B^{\sigma, p}\left(\mathbb{R}^{\ell}\right)(\sigma>0)$. If $\sigma$ is not an integer then

$$
B^{\sigma, p}\left(\mathbb{R}^{\ell}\right)=W^{\sigma, p}\left(\mathbb{R}^{\ell}\right) .
$$

If $\sigma$ is an integer the space is defined as follows. Put

$$
\Delta_{x, y} f=f(x+y)+f(x-y)-2 f(x) .
$$

Then

$$
B^{1, p}\left(\mathbb{R}^{\ell}\right)=\left\{f \in L^{p}\left(\mathbb{R}^{\ell}\right): \frac{\Delta_{x, y} f}{|y|^{1+\ell / p}} \in L^{p}\left(\mathbb{R}^{\ell} \times \mathbb{R}^{\ell}\right)\right\},
$$

with norm

$$
\|f\|_{B^{1, p}}=\|f\|_{L^{p}}+\left(\iint_{\mathbb{R}^{\ell} \times \mathbb{R}^{\ell}} \frac{\left|\Delta_{x, y} f\right|^{p}}{|y|^{\ell+p}} d x d y\right)^{1 / p}
$$


(with standard modification if $p=\infty$ ) and

$$
\begin{aligned}
B^{m, p}\left(\mathbb{R}^{\ell}\right)=\{ & f \in W^{m-1, p}\left(\mathbb{R}^{\ell}\right): \\
& \left.D_{x}^{\alpha} f \in B^{1, p}\left(\mathbb{R}^{\ell}\right) \forall \alpha \in \mathbb{N}^{\ell},|\alpha|=m-1\right\}
\end{aligned}
$$

with norm

$$
\|f\|_{B^{m, p}}=\|f\|_{W^{m-1, p}}+\left(\sum_{|\alpha|=m-1} \iint_{\mathbb{R}^{\ell} \times \mathbb{R}^{\ell}} \frac{\left|D_{x}^{\alpha} \Delta_{x, y} f\right|^{p}}{|y|^{\ell+p}} d x d y\right)^{1 / p} .
$$

We recall that the following inclusions hold ( $[27, \mathrm{p} 155])$

$$
\begin{array}{ll}
W^{m, p}\left(\mathbb{R}^{\ell}\right) \subset B^{m, p}\left(\mathbb{R}^{\ell}\right) & \text { if } p \geq 2 \\
B^{m, p}\left(\mathbb{R}^{\ell}\right) \subset W^{m, p}\left(\mathbb{R}^{\ell}\right) & \text { if } 1 \leq p \leq 2 .
\end{array}
$$

When $1<p<\infty$, the dual spaces of $W^{s, p}$ and $B^{m, p}$ are respectively denoted by $W^{-s, p^{\prime}}$ and $B^{-m, p^{\prime}}$.

The following is the main result of this section.

Theorem 3.1. Suppose that $q_{c}<q<q_{c}^{*}$ and let A be defined as in Subsection 2.1. Then there exist positive constants $c_{1}, c_{2}$, depending on $q, N, k, \kappa_{+}$, such that for any $R>1$ and any $\mu \in \mathfrak{M}_{+}\left(d_{A}\right)$ with support in $B_{R / 2}$ :

$$
\begin{aligned}
& c_{1}\|\mu\|_{B^{-s, q}\left(\mathbb{R}^{N-k}\right)}^{q} \\
& \leq \int_{D_{A, R}} \mathbb{K}[|\mu|]^{q}(x) \rho(x) d x \leq c_{2}(1+R)^{\beta}\|\mu\|_{B^{-s, q}\left(\mathbb{R}^{N-k}\right)}^{q},
\end{aligned}
$$

where $s=2-\frac{\kappa_{+}+k}{q^{\prime}}, \beta=(q+1) \kappa_{+}+k-1$ and $D_{A, R}=D_{A} \cap \Gamma_{R}$. If $q=q_{c}$ the estimate remains valid for measures $\mu$ such thatthe diameter of supp $\mu$ is sufficiently small (depending on the parameters mentioned before).

Remark. When $q \geq 2$ the norms in the Besov space may be replaced by the norms in the corresponding Sobolev spaces.

Recall the admissibility condition for a measure $\mu \in \mathfrak{M}_{+}\left(d_{A}\right)$ :

$$
\int_{D_{A, R}} \mathbb{K}[\mu]^{q}(x) \rho(x) d x<\infty \quad \forall R>0
$$

and the equivalence (see (2.27)-(2.30))

$$
\begin{aligned}
& \int_{D_{A, R}} \mathbb{K}[\mu]^{q}(x) \rho(x) d x \approx J^{A, R}(\mu) \\
& :=\int_{0}^{R} \int_{B_{R}^{\prime \prime}}\left(\int_{\mathbb{R}^{N-k}} \frac{d \mu(z)}{\left.\left(\tau^{2}+\left|x^{\prime \prime}-z\right|^{2}\right) \mid\right)^{\left(N-2+2 \kappa_{+}\right) / 2}}\right)^{q} \tau^{(q+1) \kappa_{+}+k-1} d x^{\prime \prime} d \tau,
\end{aligned}
$$


where $x=\left(x^{\prime}, x^{\prime \prime}\right) \in \mathbb{R}^{k} \times \mathbb{R}^{N-k}, \tau=\left|x^{\prime}\right|$ and $B_{R}^{\prime \prime}=\left\{x^{\prime \prime} \in \mathbb{R}^{N-k}:\left|x^{\prime \prime}\right|<R\right\}$. We denote

$$
v=N-2+2 \kappa_{+} .
$$

If $2 \kappa_{+}$is an integer, it is natural to relate (3.8) to the Poisson potential of $\mu$ in $\mathbb{R}_{+}^{n}=\mathbb{R}_{+} \times \mathbb{R}_{n-1}$ where $n=N-2+2 \kappa_{+}$. We clarify this statement below.

Assuming that $2 \leq n+k-N$, denote

$$
y=\left(y_{1}, \tilde{y}, y^{\prime \prime}\right) \in \mathbb{R}^{n}, \quad \tilde{y}=\left(y_{2}, \ldots, y_{n+k-N}\right), \quad y^{\prime \prime}=\left(y_{n+k-N+1}, \ldots, y_{n}\right) .
$$

The Poisson kernel in $\mathbb{R}_{+}^{n}=\mathbb{R}_{+} \times \mathbb{R}_{n-1}$ is given by

$$
P_{n}(y)=\gamma_{n} y_{1}|y|^{-n} \quad y_{1}>0
$$

for some $\gamma_{n}>0$, and the Poisson potential of a bounded Borel measure $\mu$ with support in

$$
\mathbf{d}:=\left\{y=\left(0, y^{\prime \prime}\right) \in \mathbb{R}^{n}: y^{\prime \prime} \in \mathbb{R}_{N-k}\right\}
$$

is

$$
\mathbb{K}_{n}[\mu](y)=\gamma_{n} y_{1} \int_{\mathbb{R}^{N-k}} \frac{d \mu(z)}{\left(y_{1}^{2}+|\tilde{y}|^{2}+\left|y^{\prime \prime}-z\right|^{2}\right)^{n / 2}} \quad \forall y \in \mathbb{R}_{+}^{n} .
$$

In particular, for $\tilde{y}=0$,

$$
\mathbb{K}_{n}[\mu]\left(y_{1}, 0, y^{\prime \prime}\right)=\gamma_{n} y_{1} \int_{\mathbb{R}^{N-k}} \frac{d \mu(z)}{\left(y_{1}^{2}+\left|y^{\prime \prime}-z\right|^{2}\right)^{n / 2}} .
$$

The integral in (3.12) is precisely the same as the inner integral in (3.8).

In fact, it will be shown that, if we set

$$
n:=\{v\}=\inf \{m \in \mathbb{N}: m \geq v\}
$$

this approach also works when $2 \kappa_{+}$is not an integer. We note that, for $n$ given by (3.13),

$$
n-N+k \geq 2 \text {, }
$$

with equality only if $k=3$ and $\kappa_{+} \leq 1 / 2$ or $k=2$ and $\kappa_{+} \in(1 / 2,1]$. Indeed,

$$
n-N+k=k+\left\{2 \kappa_{+}\right\}-2
$$

and (as $\left.\kappa_{+}>0\right)\left\{2 \kappa_{+}\right\} \geq 1$. If $k=2$ then $\kappa_{+}>1 / 2$ and consequently $\left\{2 \kappa_{+}\right\} \geq 2$. These facts imply our assertion.

We also note that $\kappa_{+}$is strictly increasing relative to $\lambda_{A}$ and

$$
\kappa_{+} \begin{cases}=1 & \text { if } D_{A}=\mathbb{R}_{+}^{N} \\ <1 & \text { if } D_{A} \subsetneq \mathbb{R}_{+}^{N} \\ >1 & \text { if } D_{A} \supsetneqq \mathbb{R}_{+}^{N} .\end{cases}
$$


Finally we observe that $\gamma:=\lambda_{A}-(N-k) \kappa_{+}>0$ (see (2.15)) and, by (2.11) and (2.32),

$$
\gamma=\kappa_{+}^{2}+(k-2) \kappa_{+}, \quad q_{c}^{*}=1+\frac{-(k-2)+\sqrt{(k-2)^{2}+4 \gamma}}{\gamma} .
$$

Therefore $q_{c}^{*}$ is strictly decreasing relative to $\gamma$ and consequently also relative to $\kappa_{+}$.

The proof of the theorem is based on the following important result proved in $[28,1.14 .4$.]

Proposition 3.2. Let $1<q<\infty$ and $s>0$. Then for any bounded Borel measure $\mu$ in $\mathbb{R}^{n-1}$ there holds

$$
I(\mu)=\int_{\mathbb{R}_{+}^{n}}\left|\mathbb{K}_{n}[\mu](y)\right|^{q} e^{-y_{1}} y_{1}^{s q-1} d y \approx\|\mu\|_{B^{-s, q}\left(\mathbb{R}^{n-1}\right)}^{q} .
$$

In the first part of the proof we derive inequalities comparing $I(\mu)$ and $J^{A, R}(\mu)$. Actually, it is useful to consider a slightly more general expression than $I(\mu)$, namely:

$$
I_{\nu, \sigma}^{m, j}(\mu):=\int_{\mathbb{R}_{+}^{m+j}}\left|\int_{\mathbb{R}^{m}} \frac{y_{1} d \mu(z)}{\left(y_{1}^{2}+|\widetilde{y}|^{2}+\left|y^{\prime \prime}-z\right|^{2}\right)^{v / 2}}\right|^{q} e^{-y_{1}} y_{1}^{\sigma q-1} d y,
$$

where $v$ is an arbitrary number such that $\nu>m, j \geq 1$ and $\sigma>0$. A point $y \in \mathbb{R}_{+}^{m+j}$ is written in the form $y=\left(y_{1}, \tilde{y}, y^{\prime \prime}\right) \in \mathbb{R}_{+} \times \mathbb{R}^{j-1} \times \mathbb{R}^{m}$. We assume that $\mu$ is supported in $\mathbb{R}^{m}$. Note that,

$$
I(\mu)=\gamma_{n}^{q} I_{n, s}^{m, j} \text { where } m=N-k, \quad j=n-m=n-N+k .
$$

Put

$$
F_{\nu, m}[\mu](\tau):=\int_{\mathbb{R}^{m}}\left|\int_{\mathbb{R}^{m}} \frac{d \mu(z)}{\left(\tau^{2}+\left|y^{\prime \prime}-z\right|^{2}\right)^{\nu / 2}}\right|^{q} d y^{\prime \prime} \quad \forall \tau \in[0, \infty) .
$$

With this notation, if $j \geq 2$ then

$$
I_{v, \sigma}^{m, j}(\mu):=\int_{0}^{\infty} \int_{\mathbb{R}^{j-1}} F_{\nu, m}[\mu]\left(\sqrt{y_{1}^{2}+|\tilde{y}|^{2}}\right) e^{-y_{1}} y_{1}^{(\sigma+1) q-1} d \tilde{y} d y_{1}
$$

and if $j=1$

$$
I_{v, \sigma}^{m, 1}(\mu):=\int_{0}^{\infty} F_{\nu, m}[\mu]\left(y_{1}\right) e^{-y_{1}} y_{1}^{(\sigma+1) q-1} d y_{1}
$$


Lemma 3.3. Assume that $m<v, 0<\sigma, 2 \leq j$ and $1<q<\infty$. Then there exists a positive constant c, depending on $m, j, v, \sigma, q$, such that, for every bounded Borel measure $\mu$ with support in $\mathbb{R}^{m}$ :

$$
\frac{1}{c} \int_{0}^{\infty} F_{\nu, m}[\mu](\tau) h_{\sigma, j}(\tau) d \tau \leq I_{v, \sigma}^{m, j}(\mu) \leq c \int_{0}^{\infty} F_{\nu, m}[\mu](\tau) h_{\sigma, j}(\tau) d \tau,
$$

where $F_{v, m}$ is given by (3.20) and, for every $\tau>0$,

$$
h_{\sigma, j}(\tau)= \begin{cases}\frac{\tau^{(\sigma+1) q+j-2}}{(1+\tau)^{(\sigma+1) q}}, & \text { if } j \geq 2, \\ e^{-\tau} \tau^{(\sigma+1) q-1}, & \text { if } j=1 .\end{cases}
$$

Proof. There is nothing to prove in the case $j=1$. Therefore we assume that $j \geq 2$.

We use the notation $y=\left(y_{1}, \tilde{y}, y^{\prime \prime}\right) \in \mathbb{R} \times \mathbb{R}^{j-1} \times \mathbb{R}^{m}$. The integrand in (3.21) depends only on $y_{1}$ and $\rho:=|\widetilde{y}|$. Therefore, $I_{v, \sigma}^{m, j}$ can be written in the form

$$
I_{\nu, \sigma}^{m, j}(\mu)=c_{m, j} \int_{0}^{\infty} \int_{0}^{\infty} F_{\nu, m}[\mu]\left(\sqrt{y_{1}^{2}+\rho^{2}}\right) e^{-y_{1}} y_{1}^{(\sigma+1) q-1} d y_{1} \rho^{j-2} d \rho .
$$

We substitute $y_{1}=\left(\tau^{2}-\rho^{2}\right)^{1 / 2}$, then change the order of integration and finally substitute $\rho=r \tau$. This yields,

$$
\begin{aligned}
& c_{m, j}^{-1} I_{v, \sigma}^{m, j}(\mu) \\
& =\int_{0}^{\infty} \int_{\rho}^{\infty} F_{v, m}[\mu](\tau) \rho^{j-2} e^{-\sqrt{\tau^{2}-\rho^{2}}}\left(\tau^{2}-\rho^{2}\right)^{(\sigma+1) q / 2-1} \tau d \tau d \rho \\
& =\int_{0}^{\infty} \int_{0}^{\tau} F_{v, m}[\mu](\tau) \rho^{j-2} e^{-\sqrt{\tau^{2}-\rho^{2}}}\left(\tau^{2}-\rho^{2}\right)^{(\sigma+1) q / 2-1} \tau d \rho d \tau \\
& =\int_{0}^{\infty} \int_{0}^{1} F_{v, m}[\mu](\tau) \tau^{j-2+(\sigma+1) q} e^{-\tau \sqrt{1-r^{2}}} f(r) d r d \tau,
\end{aligned}
$$

where

$$
f(r)=r^{j-2}\left(1-r^{2}\right)^{(\sigma+1) q / 2-1} .
$$

We denote

$$
I_{\sigma}^{j}(\tau)=\int_{0}^{1} e^{-\tau \sqrt{1-r^{2}}} f(r) d r
$$

so that

$$
I_{\nu, \sigma}^{m, j}(\mu)=c_{m, j} \int_{0}^{\infty} F_{\nu, m}[\mu](\tau) \tau^{j-2+(\sigma+1) q} I_{\sigma}^{j}(\tau) d \tau
$$


To complete the proof we estimate $I_{\sigma}^{j}$. Since $j \geq 2, f \in L^{1}(0,1)$ and $I_{\sigma}^{j}$ is continuous in $[0, \infty)$ and positive everywhere. Hence, for every $\alpha>0$, there exists a positive constant $c_{\alpha}=c_{\alpha}(\sigma)$ such that

$$
\frac{1}{c_{\alpha}} \leq I_{\sigma}^{j} \leq c_{\alpha} \text { in }[0, \alpha) \text {. }
$$

Next we estimate $I_{\sigma}^{j}$ for large $\tau$. Since $j \geq 2$,

$$
I_{\sigma}^{j} \leq 2^{(\sigma+1) q / 2-1} \int_{0}^{1}(1-r)^{(\sigma+1) q / 2-1} e^{-\tau \sqrt{1-r}} d r .
$$

Substituting $r=1-t^{2}$ we obtain,

$$
I_{\sigma}^{j} \leq 2^{(\sigma+1) q / 2} \int_{0}^{1} t^{(\sigma+1) q-1} e^{-t \tau} d t=c(\sigma, q) \tau^{-(\sigma+1) q} .
$$

On the other hand, if $\tau \geq 2$,

$$
\begin{aligned}
I_{\sigma}^{j}(\tau) & =\int_{0}^{1}\left(1-t^{2}\right)^{(j-3) / 2} t^{(\sigma+1) q-1} e^{-\tau t} d t \\
& =\tau^{-(\sigma+1) q} \int_{0}^{\tau}\left(1-(s / \tau)^{2}\right)^{(j-3) / 2} s^{(\sigma+1) q-1} e^{-s} d s \\
& \geq \tau^{-(\sigma+1) q} 2^{-(j-3)} \int_{0}^{1} s^{(\sigma+1) q-1} e^{-s} d s .
\end{aligned}
$$

Combining (3.25) with (3.26)-(3.28) we obtain (3.23).

Next we derive an estimate in which integration over $\mathbb{R}_{+}^{n}=\mathbb{R}_{+}^{j} \times \mathbb{R}^{m}$ is replaced by integration over a bounded domain, for measures supported in a fixed bounded subset of $\mathbb{R}^{m}$.

Let $B_{R}^{j}(0)$ and $B_{R}^{\dot{m}}(0)$ denote the balls of radius $R$ centered at the origin, in $\mathbb{R}^{j}$ and $\mathbb{R}^{m}$ respectively. Denote

$$
F_{v, m}^{R}[\mu](\tau)=\int_{B_{R}^{m}}\left|\int_{\mathbb{R}^{m}} \frac{d \mu(z)}{\left(\tau^{2}+\left|y^{\prime \prime}-z\right|^{2}\right)^{v / 2}}\right|^{q} d y^{\prime \prime} \quad \forall \tau \in[0, \infty)
$$

and, if $j \geq 2$,

$$
I_{v, \sigma}^{m, j}(\mu ; R)=\int_{B_{R}^{j} \cap\left\{0<y_{1}\right\}} F_{v, m}^{R}[\mu]\left(\sqrt{y_{1}^{2}+|\tilde{y}|^{2}}\right) e^{-y_{1}} y_{1}^{\sigma q-1} d \tilde{y} d y_{1},
$$

where $\left(y_{1}, \tilde{y}\right) \in \mathbb{R} \times \mathbb{R}^{j-1}$. If $j=1$ we denote

$$
I_{\nu, \sigma}^{m, 1}(\mu ; R)=\int_{0}^{R} F_{v, m}^{R}[\mu]\left(y_{1}\right) e^{-y_{1}} y_{1}^{\sigma q-1} d y_{1} .
$$

Similarly to Lemma 3.3 we obtain the following: 
Lemma 3.4. If $j \geq 1$, there exists a positive constant $c$ such that, for any bounded Borel measure $\mu$ with support in $\mathbb{R}^{m} \cap B_{R}$

$$
c^{-1} \int_{0}^{R} F_{v, m}^{R}[\mu](\tau) h_{\sigma, j}(\tau) d \tau \leq I_{v, \sigma}^{m, j}(\mu ; R) \leq c \int_{0}^{R} F_{v, m}^{R}[\mu](\tau) h_{\sigma, j}(\tau) d \tau
$$

with $h_{\sigma, j}$ as in (3.24).

Proof. In the case $j=1$ there is nothing to prove. Therefore we assume that $j \geq 2$. From (3.30) we obtain

$$
I_{\nu, \sigma}^{m, j}(\mu ; R)=c_{m, j} \int_{0}^{R} \int_{0}^{\sqrt{R^{2}-\rho^{2}}} F_{\nu, m}^{R}[\mu]\left(\sqrt{y_{1}^{2}+\rho^{2}}\right) e^{-y_{1}} y_{1}^{(\sigma+1) q-1} d y_{1} \rho^{j-2} d \rho .
$$

Substituting $y_{1}=\left(\tau^{2}-\rho^{2}\right)^{1 / 2}$, then changing the order of integration and finally substituting $\rho=r \tau$ we obtain

$$
c_{m, j}^{-1} I_{\nu, \sigma}^{m, j}(\mu ; R)=\int_{0}^{R} \int_{0}^{1} F_{\nu, \mu}^{R}[\mu](\tau) \tau^{j-2+(\sigma+1) q} e^{-\tau \sqrt{1-r^{2}}} f(r) d r d \tau .
$$

where

$$
f(r)=r^{j-2}\left(1-r^{2}\right)^{(\sigma+1) q / 2-1} .
$$

The remaining part of the proof is the same as for Lemma 3.3.

Lemma 3.5. Let $1<q, 0<\sigma$ and assume that $m<v q$ and $0 \leq j-1<v$. Then there exists a positive constant $\bar{c}$, depending on $j, m, q, \sigma, v$, such that, for every $R \geq 1$ and every bounded Borel measure $\mu$ with support in $B_{R / 2}(0) \cap \mathbb{R}^{m}$,

$$
\begin{aligned}
\mid \int_{0}^{\infty} F_{v, m}[\mu](\tau) h_{\sigma, j}(\tau) d \tau & -\int_{0}^{R} F_{v, m}^{R}[\mu](\tau) h_{\sigma, j}(\tau) d \tau \mid \\
& \leq \bar{c} R^{(\sigma+1-v) q+m+j-1}\|\mu\|_{\mathfrak{M}}^{q}
\end{aligned}
$$

with $h_{\sigma, j}$ as in (3.24).

Proof. We estimate

$$
\begin{aligned}
& \left|\int_{0}^{\infty} F_{\nu, m}[\mu](\tau) h_{\sigma, j}(\tau) d \tau-\int_{0}^{R} F_{\nu, m}^{R}[\mu](\tau) h_{\sigma, j}(\tau) d \tau\right| \leq \\
& \int_{R}^{\infty}\left|F_{\nu, m}[\mu]\right|(\tau) h_{\sigma, j}(\tau) d \tau+\int_{0}^{R}\left|F_{\nu, m}[\mu]-F_{\nu, m}^{R}[\mu]\right|(\tau) h_{\sigma, j}(\tau) d \tau .
\end{aligned}
$$

For every $\tau>0$,

$$
\left|F_{v, m}[\mu]\right|(\tau) \leq \tau^{-v q}\|\mu\|_{\mathfrak{M}}^{q}
$$


Since $j-1<v q$, it follows that

$$
\begin{aligned}
\int_{R}^{\infty}\left|F_{v, m}[\mu]\right|(\tau) h_{\sigma, j}(\tau) d \tau & \leq\|\mu\|_{\mathfrak{M}}^{q} \int_{R}^{\infty} \tau^{-v q} h_{\sigma, j}(\tau) d \tau \\
& \leq c(\sigma, q)\|\mu\|_{\mathfrak{M}}^{q} \int_{R}^{\infty} \frac{\tau^{(\sigma+1) q+j-2-v q}}{(1+\tau)^{(\sigma+1) q}} d \tau \\
& \leq \frac{c(\sigma, q)}{v q-j+1}\|\mu\|_{\mathfrak{M}}^{q} R^{j-1-v q}
\end{aligned}
$$

Since, by assumption, supp $\mu \subset B_{R / 2}$, we have

$$
\begin{aligned}
\int_{0}^{R} & \left|F_{\nu, m}[\mu]-F_{\nu, m}^{R}[\mu]\right|(\tau) h_{\sigma, j}(\tau) d \tau \\
& \leq \int_{0}^{R} \int_{\left|y^{\prime \prime}\right|>R}\left|\int_{\mathbb{R}^{m}} \frac{d \mu(z)}{\left(\tau^{2}+\left|y^{\prime \prime}-z\right|^{2}\right)^{v / 2}}\right|^{q} d y^{\prime \prime} h_{\sigma, j}(\tau) d \tau \\
& \leq\|\mu\|_{\mathfrak{M}}^{q} \int_{0}^{R} \int_{|\zeta|>R / 2}\left(\left.\left|\tau^{2}+\right| \zeta\right|^{2}\right)^{-v q / 2} d \zeta h_{\sigma, j} d \tau \\
& \leq c(m, q)\|\mu\|_{\mathfrak{M}}^{q} \int_{0}^{R} \int_{R / 2}^{\infty}\left(\tau^{2}+\rho^{2}\right)^{-v q / 2} \rho^{m-1} d \rho h_{\sigma, j} d \tau \\
& \leq c(m, q)\|\mu\|_{\mathfrak{M}}^{q} \int_{0}^{R} \tau^{m-v q} \int_{R / 2 \tau}^{\infty}\left(1+\eta^{2}\right)^{-v q / 2} \eta^{m-1} d \eta h_{\sigma, j} d \tau \\
& \leq \frac{c(m, q)}{v q-m}\|\mu\|_{\mathfrak{M}}^{q} R^{m-v q} \int_{0}^{R} \tau^{(\sigma+1) q+j-2} d \tau \\
& \leq \frac{c(m, q)}{(v q-m)((\sigma+1) q+j-1)}\|\mu\|_{\mathfrak{M}}^{q} R^{(\sigma+1) q+j-1+m-v q} .
\end{aligned}
$$

Combining (3.34)-(3.37) we obtain (3.33).

Corollary 3.6. For every $R>0$ put

$$
J_{v, \sigma}^{m, j}(\mu ; R):=\int_{0}^{R} F_{v, m}^{R}[\mu](\tau) \tau^{(\sigma+1) q+j-2} d \tau .
$$

Then

$$
\begin{aligned}
\frac{1}{c} I_{\nu, \sigma}^{m, j}(\mu)-\bar{c} R^{\beta}\|\mu\|_{\mathfrak{M}}^{q} & \leq J_{\nu, \sigma}^{m, j}(\mu ; R) \leq c R^{(\sigma+1) q} I_{\nu, \sigma}^{m, j}(\mu), \\
\beta & =(\sigma+1-\nu) q+j+m-1,
\end{aligned}
$$

for every $R>1$ and every bounded Borel measure $\mu$ with support in $B_{R / 2}^{m}(0):=$ $B_{R / 2}(0) \cap \mathbb{R}^{m}$. 
Proof. This is an immediate consequence of Lemma 3.5 and Lemma 3.3.

Lemma 3.7. Let $m, j$ be positive integers such that $j \geq 1$ and let $1<q, 0<\sigma$. Put $n:=m+j$.

Then there exist positive constants $c, \bar{c}$, depending on $j, m, q, \sigma$, such that, for every $R>1$ and every measure $\mu \in \mathfrak{M}_{+}\left(B_{R / 2}^{m}(0)\right)$,

$$
\begin{aligned}
& \frac{1}{c}\|\mu\|_{B^{-\sigma, q}\left(\mathbb{R}^{n-1}\right)}^{q}-\bar{c} R^{q\left(\sigma-\frac{n-1}{q^{\prime}}\right)}\|\mu\|_{\mathfrak{M}}^{q} \leq J_{n, \sigma}^{m, j}(\mu ; R) \\
& \leq c R^{(\sigma+1) q}\|\mu\|_{B^{-\sigma, q}\left(\mathbb{R}^{n-1}\right)}^{q} .
\end{aligned}
$$

If $\sigma<\frac{n-1}{q^{\prime}}$, there exists $R_{0}>1$ such that, for all $R>R_{0}$,

$$
\frac{1}{2 c}\|\mu\|_{B^{-\sigma, q}\left(\mathbb{R}^{n-1}\right)}^{q} \leq J_{n, \sigma}^{m, j}(\mu ; R) .
$$

If $\sigma=\frac{n-1}{q^{\prime}}$ then, there exists $a>0$ such thatthe inequality remains valid for measures $\mu$ such thatdiam $(\operatorname{supp} \mu) \leq a$.

If, in addition, $\frac{j-1}{q^{\prime}}<\sigma$ then

$$
\frac{1}{2 c}\|\mu\|_{B^{-s, q}\left(\mathbb{R}^{m}\right)}^{q} \leq J_{n, \sigma}^{m, j}(\mu ; R) \leq c R^{(\sigma+1) q}\|\mu\|_{B^{-s, q}\left(\mathbb{R}^{m}\right)}^{q}
$$

where $s:=\sigma-\frac{j-1}{q^{\prime}}$.

Remark. Assume that $\mu \geq 0$. Then:

(i) If $\mu \in B^{-\sigma, q}\left(\mathbb{R}^{n-1}\right)$ and $\frac{j-1}{q^{\prime}} \geq \sigma$ then $\mu\left(\mathbb{R}^{m}\right)=0$.

(ii) If $\mu \in B^{-s, q}\left(\mathbb{R}^{m}\right)$ and $\sigma>(n-1) / q^{\prime}$ then $s>m / q^{\prime}$ and therefore $B^{s, q^{\prime}}\left(\mathbb{R}^{m}\right)$ can be embedded in $C\left(\mathbb{R}^{m}\right)$.

Proof. Inequality (3.40) follows from (3.39) and Proposition 3.2 (see also (3.19)). For positive measures $\mu$,

$$
\|\mu\|_{\mathfrak{M}}=\mu\left(\mathbb{R}^{n-1}\right) \leq\|\mu\|_{B^{-\sigma, q}\left(\mathbb{R}^{n-1}\right)}^{q} .
$$

Therefore, if $\sigma<\frac{n-1}{q^{\prime}},(3.40)$ implies that there exists $R_{0}>1$ such that(3.41) holds for all $R>R_{0}$.

If $\sigma=\frac{n-1}{q^{\prime}}(3.40)$ implies that

$$
\frac{1}{c}\|\mu\|_{B^{-\sigma, q}\left(\mathbb{R}^{n-1}\right)}^{q}-\bar{c}\|\mu\|_{\mathfrak{M}}^{q} \leq J_{n, \sigma}^{m, j}(\mu ; R) .
$$

But if $\mu$ is a positive bounded measure such thatdiam $(\operatorname{supp} \mu) \leq a$ then

$$
\|\mu\|_{\mathfrak{M}} /\|\mu\|_{B^{-\sigma, q}\left(\mathbb{R}^{n-1}\right)}^{q} \rightarrow 0 \text { as } a \rightarrow 0 .
$$


The last inequality follows from the imbedding theorem for Besov spaces according to which there exists a continuous trace operator $T: B^{\sigma, q^{\prime}}\left(\mathbb{R}^{n-1}\right) \mapsto B^{s, q^{\prime}}\left(\mathbb{R}^{m}\right)$ and a continuous lifting $T^{\prime}: B^{s, q^{\prime}}\left(\mathbb{R}^{m}\right) \mapsto B^{\sigma, q^{\prime}}\left(\mathbb{R}^{n-1}\right)$ where $s=\sigma-\frac{n-m-1}{q^{\prime}}$.

$$
\begin{aligned}
& \text { If } v \in \mathbb{N} \text { and } \sigma=s+\frac{\nu-m-1}{q^{\prime}} \\
& \qquad \begin{aligned}
J_{v, \sigma}^{m, \nu-m}(\mu ; R) & =\int_{0}^{R} F_{\nu, m}^{R}[\mu](\tau) \tau^{(\sigma+1) q+\nu-m-2} d \tau \\
& =\int_{0}^{R} F_{v, m}^{R}[\mu](\tau) \tau^{(s+\nu-m) q-1} d \tau .
\end{aligned}
\end{aligned}
$$

However, if $\mu$ is positive, the expression

$$
M_{\nu, s}^{m}(\mu ; R):=\int_{0}^{R} F_{\nu, m}^{R}[\mu](\tau) \tau^{(s+\nu-m) q-1} d \tau,
$$

is meaningful for any real $v>m$ and $s>0$. Furthermore, as shown below, the results stated in Lemma 3.7 can be extended to this general case.

Theorem 3.8. Let $1<q, v \in \mathbb{R}$ and $m$ a positive integer. Assume that $1 \leq v-m$ and $0<s<m / q^{\prime}$. Then there exists a positive constant $c$ such that, for every bounded positive measure $\mu$ supported in $\mathbb{R}^{m} \cap B_{R / 2}(0), R>1$,

$$
\frac{1}{c}\|\mu\|_{B^{-s, q}\left(\mathbb{R}^{m}\right)}^{q} \leq M_{v, s}^{m}(\mu ; R) \leq c R^{(s+\nu-m) q+1}\|\mu\|_{B^{-s, q}\left(\mathbb{R}^{m}\right)}^{q} .
$$

This also holds when $s=m / q^{\prime}$, provided that the diameter of $\operatorname{supp} \mu$ is sufficiently small.

Proof. If $v$ is an integer and $j:=v-m$ then this statement is part of Lemma 3.7. Indeed the condition $s>0$ means that $\sigma=s+\frac{j-1}{q^{\prime}}>\frac{j-1}{q^{\prime}}$ and the condition $s<m / q^{\prime}$ means that $\sigma<\frac{n-1}{q^{\prime}}$.

Therefore we assume that $v \notin \mathbb{N}$. Let $n:=\{v\}$ and $\theta:=n-v$ so that $0<\theta<1$. Our assumptions imply that $1 \leq n-m-1$ because (as $v$ is not an integer) $v-m>1$ and consequently $n-m \geq 2$.

If $a, b$ are positive numbers, put

$$
A_{v}:=\frac{a^{(s+v-m) q-1}}{\left(a^{2}+b^{2}\right)^{v q / 2}} .
$$

Obviously $A_{v}$ decreases as $v$ increases. Therefore, $A_{n} \leq A_{v} \leq A_{n-1}$ which in turn implies,

$$
M_{n, s}^{m} \leq M_{v, s}^{m} \leq M_{n-1, s}^{m} .
$$

By Lemma 3.7, the assertions of the theorem are valid in the case that $v=n$ or $v=n-1$. Therefore the previous inequality implies that the assertions hold for any real $v$ subject to the conditions imposed. 
By (3.8),

$$
J^{A, R}=\int_{0}^{R} F_{\nu, m}^{R}(\tau) \tau^{(q+1) \kappa_{+}+k-1} d \tau,
$$

where $m=N-k$ and $v=N-2+2 \kappa_{+}$. Consequently, by (3.38),

$$
J^{A, R}=M_{v, s}^{m}
$$

where $s$ is determined by,

$$
(s+v-m) q-1=(q+1) \kappa_{+}+k-1, \quad k=v-m+2-2 \kappa_{+} .
$$

It follows that

$$
s q=-\left(k-2+2 \kappa_{+}\right) q+(q+1) \kappa_{+}+k=k(1-q)+2 q-\kappa_{+}(q-1)
$$

and therefore

$$
s=2-\frac{k+\kappa_{+}}{q^{\prime}} .
$$

Proof of Theorem 3.1. Put

$$
v:=N-2+2 \kappa_{+}, \quad s:=2-\frac{\kappa_{+}+k}{q^{\prime}}, \quad m:=N-k .
$$

Recall that in the case $k=2$ we have $\kappa_{+}>1 / 2$. Therefore

$$
v-m-1=k-3+2 \kappa_{+}>0 .
$$

Furthermore,

$$
(s+v-m) q-1=(q+1) \kappa_{+}+k-1, \quad k=v-m+2-2 \kappa .
$$

Thus

$$
J^{A, R}=\int_{0}^{R} F_{v, m}^{R}(\tau) \tau^{(q+1) \kappa_{+}+k-1} d \tau=M_{v, s}^{m} .
$$

Next we show that $0<s \leq m / q^{\prime}$. More precisely we prove

$$
0<s \leq m / q^{\prime} \Longleftrightarrow q_{c} \leq q<q_{c}^{*} .
$$

Let $\mu$ be a bounded non-negative Borel measure in $B^{-s, q}\left(\mathbb{R}^{m}\right)$. If $s \leq 0$, $B^{-s, q}\left(\mathbb{R}^{m}\right) \subset L^{q}\left(\mathbb{R}^{m}\right)$. Therefore, in this case, every bounded Borel measure on $\mathbb{R}^{m}$ is admissible i.e. satisfies (2.35). Consequently, by Proposition 2.2, $q<q_{c}$. As we assume $q \geq q_{c}$ it follows that $s>0$.

If $s>0$ and $s q^{\prime}-m \geq 0$ then $C_{s, q^{\prime}}(K)=0$ for every compact subset of $\mathbb{R}^{m}$ and consequently $\mu(K)=0$ for any such set. Conversely, if $s q^{\prime}-m<0$ then there exist non-trivial positive bounded measures in $B^{-s, q}\left(\mathbb{R}^{m}\right)$. Therefore, by Proposition 2.1,sq $<m$ if and only if $q<q_{c}^{*}$.

In conclusion, $0<s \leq m / q^{\prime}$ and $v-m \geq 1$; therefore Theorem 3.1 is a consequence of Theorem 3.8 . 
Remark. Note that the critical exponent for the imbedding of $B^{2-\frac{\kappa_{+}+k}{q^{\prime}}, q^{\prime}}\left(\mathbb{R}^{N-k}\right)$ into $C\left(\mathbb{R}^{N-k}\right)$ is again

$$
q=q_{c}=\frac{N+\kappa_{+}}{N+\kappa_{+}-2} .
$$

\section{Supercritical equations in a polyhedral domain}

In this section $q$ is a real number larger than 1 and $P$ an $N$-dim polyhedral domain as described in Subsection 6.1. Denote by $\left\{L_{k, j}: k=1, \ldots, N, j=1, \ldots, n_{k}\right\}$ the family of faces, edges and vertices of $P$. In this notation, $L_{1, j}$ denotes one of the open faces of $P$; for $k=2, \ldots, N-1, L_{k, j}$ denotes a relatively open $(N-k)$-dimensional edge and $L_{N, j}$ denotes a vertex. For $1 \leq k<N$, the $(N-k)$ dimensional space which contains $L_{k, j}$ is denoted by $\mathbb{R}_{j}^{N-k}$. If $1<k<N$, the cylinder of radius $r$ around the axis $\mathbb{R}_{j}^{N-k}$ will be denoted by $\Gamma_{k, j, r}^{\infty}$ and the subset $A_{k, j}$ of $S^{k-1}$ is defined by

$$
\lim _{r \rightarrow 0} \frac{1}{r}\left(\partial \Gamma_{k, j, r}^{\infty} \cap P\right)=L_{k, j} \times A_{k, j} .
$$

$A_{k, j}$ is the 'opening' of $P$ at the edge $L_{k, j}$. For $k=N$ we replace in this definition the cylinder $\Gamma_{N, j, r}^{\infty}$ by the ball $B_{r}\left(L_{N, j}\right)$. For $1<k \leq N$ and $A=A_{k, j}$ we use $d_{A}$ as an alternative notation for $\mathbb{R}_{j}^{N-k}$ and denote by $D_{A}$ the $k$-dihedron with edge $d_{A}$ and opening $A$ as in Subsection 6.1 (with $S_{A}$ defined as in (2.2)). For $k=1, D_{A}$ stands for the half space $\mathbb{R}_{j}^{N-1} \times(0, \infty)$.

\subsection{Definitions and auxiliary results}

Let $\Omega$ be a bounded Lipschitz domain. We say that $\left\{\Omega_{n}\right\}$ is a Lipschitz exhaustion of $\Omega$ if, for every $n, \Omega_{n}$ is Lipschitz and

$$
\Omega_{n} \subset \bar{\Omega}_{n} \subset \Omega_{n+1}, \quad \Omega=\cup \Omega_{n}, \quad \mathbb{H}_{N-1}\left(\partial \Omega_{n}\right) \rightarrow \mathbb{H}_{N-1}(\partial \Omega) .
$$

If $\omega_{n}$ (respectively $\omega$ ) is the harmonic measure in $\Omega_{n}$ (respectively $\Omega$ ) relative to $x_{0} \in \Omega_{1}$, then, for every $Z \in C(\bar{\Omega})$,

$$
\lim _{n \rightarrow \infty} \int_{\partial \Omega_{n}} Z d \omega_{n}=\int_{\partial \Omega} Z d \omega .
$$

[24, Lemma 2.1]. Furthermore, if $\mu$ is a bounded Borel measure on $\partial \Omega$ and $v:=$ $\mathbb{K}^{\Omega}[\mu]$, there holds

$$
\lim _{n \rightarrow \infty} \int_{\partial \Omega_{n}} Z v d \omega_{n}=\int_{\partial \Omega} Z d \mu,
$$

[24, Lemma 2.2]. If $v$ is a positive solution and (4.3) holds we say that $\mu$ is the boundary trace of $v$.

The following estimates are proved in [24, Lemma 2.3]: 
Proposition 4.1. Let $\mu$ be bounded Borel measures on $\partial \Omega$. Then $\mathbb{K}[\mu] \in L_{\rho}^{1}(\Omega)$ and there exists a constant $C=C(\Omega)$ such that

$$
\|\mathbb{K}[\mu]\|_{L_{\rho}^{1}(\Omega)} \leq C\|\mu\|_{\mathfrak{M}(\partial \Omega)} .
$$

In particular if $h \in L^{1}(\partial \Omega ; \omega)$ then

$$
\|\mathbb{P}[h]\|_{L_{\rho}^{1}(\Omega)} \leq C\|h\|_{L^{1}(\partial \Omega ; \omega)} .
$$

The nest result will be used in deriving estimates in a $k$-dimensional dihedron when the boundary data is concentrated on the edge.

Proposition 4.2. We denote by $G^{\Omega_{n}}$ (respectively $G^{\Omega}$ ) the Green function in $\Omega_{n}$ (respectively $\Omega$ ). Let $v$ be a positive harmonic function in $\Omega$ with boundary trace $\mu$. Let $Z \in C^{2}(\bar{\Omega})$ and let $\tilde{G} \in C^{\infty}(\Omega)$ be a function that coincides with $x \mapsto G\left(x, x_{0}\right)$ in $Q \cap \Omega$ for some neighborhood $Q$ of $\partial \Omega$ and some fixed $x_{0} \in \Omega$. In addition assume that there exists a constant $c>0$ such that

$$
|\nabla Z \cdot \nabla \tilde{G}| \leq c \rho .
$$

Under these assumptions, if $\zeta:=Z \tilde{G}$ then

$$
-\int_{\Omega} v \Delta \zeta d x=\int_{\partial \Omega} Z d \mu
$$

Proof. Let $\left\{\Omega_{n}\right\}$ be a $C^{1}$ exhaustion of $\Omega$. We assume that $\partial \Omega_{n} \subset Q$ for all $n$ and $x_{0} \in \Omega_{1}$. Let $\tilde{G}_{n}(x)$ be a function in $C^{1}\left(\Omega_{n}\right)$ such that $\tilde{G}_{n}$ coincides with $G^{\Omega_{n}}\left(\cdot, x_{0}\right)$ in $Q \cap \Omega_{n}, \tilde{G}_{n}\left(\cdot, x_{0}\right) \rightarrow \tilde{G}\left(\cdot, x_{0}\right)$ in $C^{2}(\Omega \backslash Q)$ and $\tilde{G}_{n}\left(\cdot, x_{0}\right) \rightarrow \tilde{G}\left(\cdot, x_{0}\right)$ in $\operatorname{Lip}(\Omega)$. If $\zeta_{n}=Z \tilde{G}_{n}$ we have,

$$
\begin{aligned}
-\int_{\Omega_{n}} v \Delta \zeta_{n} d x & =\int_{\partial \Omega_{n}} v \partial_{\mathbf{n}} \zeta d S=\int_{\partial \Omega_{n}} v Z \partial_{\mathbf{n}} \tilde{G}_{n}\left(\xi, x_{0}\right) d S \\
& =\int_{\partial \Omega_{n}} v Z P^{\Omega_{n}}\left(x_{0}, \xi\right) d S=\int_{\partial \Omega_{n}} v Z d \omega_{n} .
\end{aligned}
$$

By (4.3),

$$
\int_{\partial \Omega_{n}} v Z d \omega_{n} \rightarrow \int_{\partial \Omega} Z d \mu
$$

On the other hand, in view of (4.6), we have

$$
\Delta \zeta_{n}=\tilde{G}_{n} \Delta Z+Z \Delta \tilde{G}_{n}+2 \nabla Z \cdot \nabla \tilde{G}_{n} \rightarrow \Delta Z
$$

in $L_{\rho}^{1}(\Omega)$; therefore,

$$
-\int_{\Omega_{n}} v \Delta \zeta_{n} d x \rightarrow-\int_{\Omega} v \Delta \zeta d x .
$$


We denote by $\mathfrak{M}_{q}=\mathfrak{M}_{q}(\partial \Omega)$ the set of $q$-good measures on the boundary. A positive solution $u$ of (1.1) in $\Omega$ possesses a boundary trace $\mu \in \mathfrak{M}(\partial \Omega)$ if and only if

$$
\int_{\Omega} u^{q} \rho d x<\infty
$$

[24, Proposition 4.1]. In this case $\mu \in \mathfrak{M}_{q}$.

The following statements can be proved in the same way as in the case of smooth domains. For the proof in that case see [20].

I. $\mathfrak{M}_{q}(\partial \Omega)$ is a linear space and

$$
\mu \in \mathfrak{M}_{q}(\partial \Omega) \Longleftrightarrow|\mu| \in \mathfrak{M}_{q}(\partial \Omega)
$$

II. If $\left\{\mu_{n}\right\}$ is an increasing sequence of measures in $\mathfrak{M}_{q}(\partial \Omega)$ and $\mu:=\lim \mu_{n}$ is a finite measure, then $\mu \in \mathfrak{M}_{q}(\partial \Omega)$.

Proposition 4.3. Let $\mu$ be a bounded measure on $\partial P$. ( $\mu$ may be a signed measure.) For $i=1, \ldots, N, j=1, \ldots, n_{i}$, we define the measure $\mu_{k, j}$ on $d_{A_{k, j}}$ by,

$$
\mu_{k, j}=\mu \text { on } L_{k, j}, \quad \mu_{k, j}=0 \text { on } d_{A_{k, j}} \backslash L_{k, j} .
$$

Then $\mu \in \mathfrak{M}_{q}(\partial P)$, i.e., the problem

$$
-\Delta u+u^{q}=0 \text { in } P, u=\mu \text { on } \partial P
$$

possesses a solution, if and only if $\mu_{k, j}$ is a q-good measure relative to $D_{A_{k, j}}$ for all $(k, j)$ as above.

Proof. In view of statement $\mathbf{I}$ above, it is sufficient to prove the proposition in the case that $\mu$ is non-negative. This is assumed hereafter. If $\mu \in \mathfrak{M}_{q}(\partial P)$ then any measure $v$ on $\partial P$ such that $0 \leq v \leq \mu$ is a $q$-good measure relative to $P$. Therefore

$$
\mu \in \mathfrak{M}_{q}(\partial P) \Longrightarrow \mu_{k, j}^{\prime}:=\mu \chi_{L_{k, j}} \in \mathfrak{M}_{q}(\partial P) \text {. }
$$

Assume that $\mu \in \mathfrak{M}_{q}(\partial P)$ and let $u_{k, j}$ be the solution of (4.9) when $\mu$ is replaced by $\mu_{k, j}^{\prime}$. Denote by $u_{k, j}^{\prime}$ the extension of $u_{k, j}$ by zero to the $k$-dihedron $D_{A_{k, j}}$. Then $u_{k, j}^{\prime}$ is a subsolution of (1.1) in $D_{A_{k, j}}$ with boundary data $\mu_{k, j}$. In the present case there always exists a supersolution, e.g. the maximal solution of (1.1) in $D_{A_{k, j}}$ vanishing outside $d_{A_{k, j}} \backslash \bar{L}_{k, j}$. Therefore there exists a solution $v_{k, j}$ of this equation in $D_{A_{k, j}}$ with boundary data $\mu_{k, j}$, i.e., $\mu_{k, j}$ is $q$-good relative to $D_{A_{k, j}}$.

Next assume that $\mu \in \mathfrak{M}(\partial P)$ and that $\mu_{k, j}$ is $q$-good relative to $D_{A_{k, j}}$ for every $(k, j)$ as above. Let $v_{k, j}$ be the solution of (1.1) in $D_{A_{k, j}}$ with boundary data $\mu_{k, j}$. Then $v_{k, j}$ is a supersolution of problem (4.9) with $\mu$ replaced by $\mu_{k, j}^{\prime}$ and consequently there exists a solution $u_{k, j}$ of this problem. It follows that

$$
w:=\max \left\{u_{k, j}: k=1, \ldots, N, j=1, \ldots, n_{k}\right\}
$$


is a subsolution while

$$
\bar{w}:=\sum_{\substack{k=1, \ldots, N \\ j=1, \ldots, n_{k}}} u_{k, j}
$$

is a supersolution of (4.9). Consequently there exists a solution of this problem, i.e., $\mu \in \mathfrak{M}_{q}(\partial P)$.

\subsection{Removable singular sets and 'good measures', I}

We first introduce some standard elements associated to the Bessel capacities which are the natural way to characterize good measures or removable sets. For $\alpha \in \mathbb{R}$, we denote by $G_{\alpha}$ the Bessel kernel of order $\alpha$, defined by

$$
G_{\alpha}(\xi)=\mathcal{F}^{-1}\left(\left(1+|\cdot|^{2}\right)^{-\frac{\alpha}{2}}\right)(\xi),
$$

where $\mathcal{F}$ is the Fourier transform in the space $\mathcal{S}^{\prime}\left(\mathbb{R}^{\ell}\right)$ of moderate distributions in $\mathbb{R}^{\ell}$. For $1 \leq p \leq \infty$, the Bessel space $L_{\alpha, p}\left(\mathbb{R}^{\ell}\right)$ is defined by

$$
L_{\alpha, p}\left(\mathbb{R}^{\ell}\right)=\left\{f: f=G_{\alpha} * g,: g \in L^{p}\left(\mathbb{R}^{\ell}\right)\right\},
$$

with norm

$$
\|f\|_{L_{\alpha, p}}=\|g\|_{L_{p}}=\left\|G_{-\alpha} * f\right\|_{L_{p}} .
$$

For $\alpha, \beta \in \mathbb{R}$ and $1<p<\infty$, the mapping $f \mapsto G_{\beta} * f$ is an isomorphism from $L_{\alpha, p}\left(\mathbb{R}^{\ell}\right)$ into $L_{\alpha+\beta, p}\left(\mathbb{R}^{\ell}\right)$. Finally the Bessel spaces are connected to Besov and Sobolev spaces: when $\alpha>0$ and $1<p<\infty$, it is known that if $\alpha \in \mathbb{N}$, $L_{\alpha, p}\left(\mathbb{R}^{\ell}\right)=W^{\alpha, p}\left(\mathbb{R}^{\ell}\right)$ and if $\alpha \notin \mathbb{N}$, then $L_{\alpha, p}\left(\mathbb{R}^{\ell}\right)=B^{\alpha, p}\left(\mathbb{R}^{\ell}\right)$, with equivalent norms (see e.g. [5,27]).

The Bessel capacity $C_{\alpha, p}^{\mathbb{R}^{\ell}}(\alpha>0, p \geq 1)$ is defined by the following rules: if $K \subset \mathbb{R}^{\ell}$ is compact

$$
C_{\alpha, p}^{\mathbb{R}^{\ell}}(K)=\inf \left\{\|f\|_{L_{\alpha, p}}^{p}: f \in \mathcal{S}\left(\mathbb{R}^{\ell}\right), f \geq \chi_{K}\right\}
$$

If $G$ is open

$$
C_{\alpha, p}^{\mathbb{R}^{\ell}}(G)=\sup \left\{C_{\alpha, p}^{\mathbb{R}^{\ell}}(K): K \subset G, K \text { compact }\right\}
$$

If $A$ is any set

$$
C_{\alpha, p}^{\mathbb{R}^{\ell}}(A)=\inf \left\{C_{\alpha, p}^{\mathbb{R}^{\ell}}(G): A \subset G, G \text { open }\right\}
$$

Note that the capacity of any non-empty set is positive if and only if $\alpha>\frac{\ell}{p}$ because of Sobolev-Besov embedding theorem. 
Proposition 4.4. Let $A$ be a Lipschitz domain on $S^{k-1}, 2 \leq k \leq N-1$, and let $D_{A}$ be the $k$-dihedron with opening $A$. Let $\mu \in \mathfrak{M}\left(\partial D_{A}\right)$ be a positive measure with compact support contained in $d_{A}\left(=\right.$ the edge of $\left.D_{A}\right)$. Assume that $\mu$ is $q$-good relative to $D_{A}$. Let $R>1$ be large enough so that $\operatorname{supp} \mu \subset B_{R}^{N-k}(0)$ and let $u$ be the solution of (1.1) in $D_{A}^{R}$ with trace $\mu$ on $d_{A}^{R}$ and trace zero on $\partial D_{A}^{R} \backslash d_{A}^{R}$. Then:

(i) For every non-negative $\eta \in C_{0}^{\infty}\left(B_{3 R / 4}^{N-k}(0)\right)$,

$$
\begin{aligned}
\left(\int_{d_{A}^{R}} \eta^{q^{\prime}} d \mu\right) \leq & c M^{q^{\prime}} \int_{D_{A}^{R}} u^{q} \rho d x \\
& +c M^{q^{\prime}}\left(\int_{D_{A}^{R}} u^{q} \rho d x\right)^{\frac{1}{q}}\left(1+M^{-1}\|\eta\|_{L^{q^{\prime}}\left(d_{A}^{R}\right)}\right) .
\end{aligned}
$$

where $M=\|\eta\|_{L^{\infty}}$ and $\rho$ is the first eigenfunction of $-\Delta$ in $D_{A}^{R}$ normalized by $\rho\left(x_{0}\right)=1$ at some point $x_{0} \in D_{A}^{R}$. The constant $c$ depends only on $N, q, k, x_{0}, \lambda_{1}, R$ where $\lambda_{1}$ is the first eigenvalue.

(ii) For any compact set $E \subset d_{A}$,

$$
C_{s, q}^{N-k}(E)=0 \Longrightarrow \mu(E)=0, \quad s=2-\frac{\kappa_{+}+k}{q^{\prime}},
$$

where $C_{s, q}^{N-k}$ denotes the Bessel capacity with the indicated indices in $\mathbb{R}^{N-k}$.

Remark. If we replace $D_{A}^{R}$ by $D_{A} \cap B_{\tilde{R}}^{k}(0) \cap B_{R}^{N-k}(0), \tilde{R}>1$, then the constant $c$ in (i) depends on $\tilde{R}$ but not on $R$.

Proof. We identify $d_{A}$ with $\mathbb{R}^{N-k}$ and use the notation

$$
x=\left(x^{\prime}, x^{\prime \prime}\right) \in \mathbb{R}^{k} \times \mathbb{R}^{N-k}, \quad y=\left|x^{\prime}\right| .
$$

Let $\eta \in C_{0}^{\infty}\left(\mathbb{R}^{N-k}\right)$ and let $R$ be large enough so that supp $\eta \subset B_{R / 2}^{N-k}(0)$. Let $w=w_{R}\left(t, x^{\prime \prime}\right)$ be the solution of the following problem in $\mathbb{R}_{+} \times B_{R}^{N-k}(0)$ :

$$
\begin{aligned}
\partial_{t} w-\Delta_{x^{\prime \prime}} w & =0 & & \text { in } \mathbb{R}^{+} \times B_{R}^{N-k}(0), \\
w\left(0, x^{\prime \prime}\right) & =\eta\left(x^{\prime \prime}\right) & & \text { in } B_{R}^{N-k}, \\
w\left(t, x^{\prime \prime}\right) & =0 & & \text { on } \partial B_{R}^{N-k}(0) .
\end{aligned}
$$

Thus $w_{R}(t, \cdot)=S_{R}(t)[\eta]$ where $S_{R}(t)$ is the semi-group operator corresponding to the above problem. Denote,

$$
H_{R}[\eta]\left(x^{\prime}, x^{\prime \prime}\right)=w_{R}\left(\left|x^{\prime}\right|^{2}, x^{\prime \prime}\right)=S_{R}\left(y^{2}\right)[\eta]\left(x^{\prime \prime}\right), \quad y:=\left|x^{\prime}\right| .
$$


We assume, as we may, that $R>1$. Let $\rho^{R}$ be the first eigenfunction of $-\Delta_{x^{\prime \prime}}$ in the ball $B_{R}^{N-k}(0)$ normalized by $\rho^{R}(0)=1$ and let $\rho_{A}$ be the first eigenfunction of $-\Delta_{x^{\prime}}$ in $C_{A}$ (where $C_{A}$ denotes the cone with opening $A$ in $\mathbb{R}^{k}$ ) normalized so that $\rho_{A}\left(x_{0}^{\prime}\right)=1$ at some point $x_{0}^{\prime} \in S_{A}$. Then $\rho^{R} \rho_{A}$ is the first eigenfunction of $-\Delta$ in $\left\{x \in D_{A}:\left|x^{\prime \prime}\right|<R\right\}$. Note that $\rho^{R} \leq 1$ and $\rho^{R} \rightarrow 1$ as $R \rightarrow \infty$ in $C^{2}(I)$ for any bounded set $I \subset \mathbb{R}^{N-k}$.

Let $h \in C^{\infty}(\mathbb{R})$ be a monotone decreasing function such that $h(t)=1$ for $t<1 / 2$ and $h(t)=0$ for $t>3 / 4$. Put

$$
\psi_{R}\left(x^{\prime}\right)=h\left(\left|x^{\prime}\right| / R\right)
$$

and

$$
\zeta_{R}:=\rho_{A} \psi_{R} H_{R}[\eta]^{q^{\prime}}
$$

If $\rho_{A}^{R}$ is the first eigenfunction (normalized at $\left.x_{0}\right)$ of $D_{A}^{R}:=D_{A} \cap \Gamma_{R}\left(\Gamma_{R}\right.$ as in $(2.25))$ then

$$
\rho_{A} \psi_{R} \leq c \rho_{A}^{R}
$$

and $\rho^{R} \rho_{A}^{R}$ is the first eigenfunction in $D_{A}^{R}$.

Hereafter we shall drop the index $R$ in $\zeta_{R}, H_{R}, w_{R}$ but keep it in the other notations in order to avoid confusion.

We shall verify that $\zeta \in D_{A}^{R}$. To this purpose we compute,

$$
\begin{aligned}
\Delta \zeta= & -\lambda_{1}\left(\rho_{A} \psi_{R}\right) H[\eta]^{q^{\prime}}+\left(\rho_{A} \psi_{R}\right) \Delta H[\eta]^{q^{\prime}}+2 \nabla\left(\rho_{A} \psi_{R}\right) \cdot \nabla H[\eta]^{q^{\prime}} \\
= & -\lambda_{1} \zeta+q^{\prime}\left(\rho_{A} \psi_{R}\right)(H[\eta])^{q^{\prime}-1} \Delta H[\eta] \\
& +q\left(q^{\prime}-1\right)\left(\rho_{A} \psi_{R}\right)(H[\eta])^{q^{\prime}-2}|\nabla H[\eta]|^{2} \\
& +2 q^{\prime}(H[\eta])^{q^{\prime}-1} \nabla\left(\rho_{A} \psi_{R}\right) \cdot \nabla H[\eta] .
\end{aligned}
$$

In addition,

$$
\begin{aligned}
\nabla H[\eta] & =\nabla_{x^{\prime}} H[\eta]+\nabla_{x^{\prime \prime}} H[\eta]=\partial_{y} H[\eta] \frac{x^{\prime}}{y}+\nabla_{x^{\prime \prime}} H[\eta] \\
& =2 y \partial_{t} w\left(y^{2}, x^{\prime \prime}\right) \frac{x^{\prime}}{y}+\nabla_{x^{\prime \prime}} H[\eta]\left(x^{\prime}, x^{\prime \prime}\right)
\end{aligned}
$$

and consequently (recall that $y$ stands for $\left|x^{\prime}\right|$ ),

$$
\begin{aligned}
& \nabla H[\eta] \cdot \nabla\left(\rho_{A} \psi_{R}\right) \\
& =2 \partial_{t} w\left(y^{2}, x^{\prime \prime}\right) x^{\prime} \cdot\left(\psi_{R}\left(\left|x^{\prime}\right|^{\kappa_{+}-1}\left(\kappa_{+} \frac{x^{\prime}}{y} \omega_{k}\left(x^{\prime} / y\right)+\left|x^{\prime}\right| \nabla \omega_{k}\left(x^{\prime} / y\right)\right)\right)+\rho_{A} \nabla \psi_{R}\right) \\
& =2 \kappa_{+} \partial_{t} w\left(y^{2}, x^{\prime \prime}\right)\left|x^{\prime}\right|^{\kappa_{+}} \omega_{k}\left(x^{\prime} / y\right)=2 \partial_{t} w\left(y^{2}, x^{\prime \prime}\right)\left(\kappa_{+} \rho_{A} \psi_{R}+\rho_{A} x^{\prime} \cdot \nabla \psi_{R}\right) .
\end{aligned}
$$


Since $w=w_{R}$ vanishes for $\left|x^{\prime \prime}\right|=R$ and $\eta=0$ in a neighborhood of this sphere, $\left|\partial_{t} w\left(y^{2}, x^{\prime \prime}\right)\right| \leq c \rho^{R}$. As $\psi_{R}$ vanishes for $\left|x^{\prime}\right|>3 R / 4$ we have $\rho_{A} \nabla \psi_{R} \leq c \rho_{A}^{R}$. Therefore

$$
\left|\nabla H[\eta] \cdot \nabla \rho_{A}\right| \leq c \rho^{R} \rho_{A}^{R}
$$

and, in view of (4.21),

$$
|\Delta \zeta| \leq c \rho^{R} \rho_{A}^{R}
$$

Thus $\zeta \in X\left(D_{A}^{R}\right)$ and consequently

$$
\int_{D_{A}^{R}}\left(-u \Delta \zeta+u^{q} \zeta\right) d x=-\int_{D_{A}^{R}} \mathbb{K}[\mu] \Delta \zeta d x
$$

Since $q\left(q^{\prime}-1\right) \rho_{A}(H[\eta])^{q^{\prime}-2}|\nabla H[\eta]|^{2} \geq 0$, we have

$$
\begin{aligned}
& \left|\int_{D_{A}^{R}} u \Delta \zeta d x\right| \\
& \leq \int_{D_{A}^{R}} u\left(\lambda_{1} \zeta+q^{\prime}(H[\eta])^{q^{\prime}-1}(\rho|\Delta H[\eta]|+2|\nabla \rho . \nabla H[\eta]|)\right) d x \\
& \leq \int_{D_{A}^{R}} u\left(\lambda_{1} \zeta+q^{\prime} \zeta^{1 / q}\left(\rho^{1 / q^{\prime}}|\Delta H[\eta]|+2 \rho^{-1 / q}|\nabla \rho . \nabla H[\eta]|\right)\right) d x \\
& \leq\left(\int_{D_{A}^{R}} u^{q} \zeta d x\right)^{\frac{1}{q}}\left(\lambda_{1}\left(\int_{D_{A}^{R}} \zeta d x\right)^{\frac{1}{q^{\prime}}}+q^{\prime}\|L[\eta]\|_{L^{q^{\prime}}\left(D_{A}^{R}\right)}\right)
\end{aligned}
$$

where

$$
L[\eta]=\rho^{1 / q^{\prime}}|\Delta H[\eta]|+2 \rho^{-1 / q}|\nabla \rho . \nabla H[\eta]| .
$$

By Proposition 4.2

$$
-\int_{D_{A}^{R}} \mathbb{K}[\mu] \Delta \zeta d x=\int_{d_{A}^{R}} \eta^{q^{\prime}} d \mu
$$

Therefore

$$
\begin{aligned}
\left(\int_{d_{A}^{R}} \eta^{q^{\prime}} d \mu\right) \leq & \int_{D_{A}^{R}} u^{q} \zeta d x \\
& +\left(\int_{D_{A}^{R}} u^{q} \zeta d x\right)^{\frac{1}{q}}\left(\lambda_{1}\left(\int_{D_{A}^{R}} \zeta d x\right)^{\frac{1}{q^{\prime}}}+q^{\prime}\|L[\eta]\|_{L^{q^{\prime}}\left(D_{A}^{R}\right)}\right) .
\end{aligned}
$$

Next we prove that

$$
\|L[\eta]\|_{L^{q^{\prime}}\left(D_{A}^{R}\right)} \leq C\|\eta\|_{W^{s, q^{\prime}}\left(\mathbb{R}^{N-k}\right)}
$$


starting with the estimate of the first term on the right hand side of (4.25).

$$
\begin{aligned}
\Delta H[\eta] & =\Delta_{x^{\prime}} H[\eta]+\Delta_{x^{\prime \prime}} H[\eta]=\partial_{y}^{2} H[\eta]+\frac{k-1}{y} \partial_{y} H[\eta]+\Delta_{x^{\prime \prime}} H[\eta] \\
& =2 y^{2} \partial_{t t} w\left(y^{2}, x^{\prime \prime}\right)+k \partial_{t} w\left(y^{2}, x^{\prime \prime}\right)+\Delta_{x^{\prime \prime}} H[\eta] \\
& =2 y^{2} \partial_{t t} w\left(y^{2}, x^{\prime \prime}\right)+(k+1) \partial_{t} w\left(y^{2}, x^{\prime \prime}\right) .
\end{aligned}
$$

Then

$$
\begin{aligned}
\int_{\mathbb{R}^{N}} \rho|\Delta H[\eta]|^{q^{\prime}} d x \leq & c \int_{0}^{1} \int_{\mathbb{R}^{N-k}}\left|\partial_{t t} w\left(y^{2}, x^{\prime \prime}\right)\right|^{q^{\prime}} d x^{\prime \prime} y^{\kappa_{+}+2 q^{\prime}+k-1} d y \\
& +c \int_{0}^{1} \int_{\mathbb{R}^{N-k}}\left|\partial_{t} w\left(y^{2}, x^{\prime \prime}\right)\right|^{q^{\prime}} d x^{\prime \prime} y^{\kappa_{+}+k-1} d y \\
\leq & c \int_{0}^{1} \int_{\mathbb{R}^{N-k}}\left|\partial_{t t} w\left(t, x^{\prime \prime}\right)\right|^{q^{\prime}} d x^{\prime \prime} t^{\left(\kappa_{+}+k\right) / 2+q^{\prime}} \frac{d t}{t} \\
& +c \int_{0}^{1} \int_{\mathbb{R}^{N-k}}\left|\partial_{t} w\left(t, x^{\prime \prime}\right)\right|^{q^{\prime}} d x^{\prime \prime} t^{(\kappa++k) / 2} \frac{d t}{t} \\
\leq & c \int_{0}^{1}\left\|t^{\left.2-\left(1-\frac{\kappa++k}{2 q^{\prime}}\right)\right)} \frac{d^{2} S(t)[\eta]}{d t^{2}}\right\|_{L^{q^{\prime}}\left(\mathbb{R}^{N-k}\right)}^{q^{\prime}} \frac{d t}{t} \\
& +c \int_{0}^{1}\left\|t^{1-\left(1-\frac{\kappa++k}{2 q^{\prime}}\right)} \frac{d S(t)[\eta]}{d t}\right\|_{L^{q^{\prime}\left(\mathbb{R}^{N-k}\right)}}^{q^{\prime}} \frac{d t}{t} .
\end{aligned}
$$

Put $\beta=\frac{\kappa_{+}+k}{2 q^{\prime}}$ and note that $0<\beta=\frac{1}{2}(2-s)<1$. By standard interpolation theory,

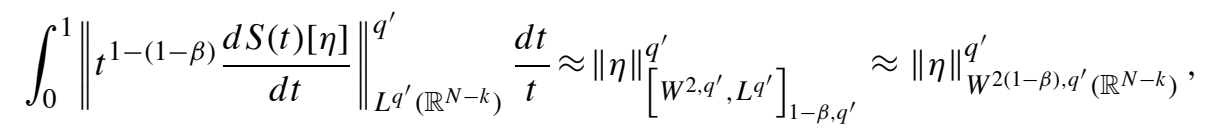

and

$$
\int_{0}^{1}\left\|t^{2-(1-\beta))} \frac{d^{2} S(t)[\eta]}{d t^{2}}\right\|_{L^{q^{\prime}\left(\mathbb{R}^{N-k}\right)}}^{q^{\prime}} \frac{d t}{t} \approx\|\eta\|_{\left[W^{4, q^{\prime}, L^{q^{\prime}}}\right]_{\frac{1}{2}(1-\beta), q^{\prime}}^{q^{\prime}}}^{\approx} \approx \eta \|_{W^{2(1-\beta), q^{\prime}\left(\mathbb{R}^{N-k}\right)}}^{q^{\prime}} .
$$


The second term on the right hand side of (4.25) is estimated in a similar way:

$$
\begin{aligned}
& \int_{\mathbb{R}^{N}} \rho^{-q^{\prime} / q}|\nabla H[\eta] \cdot \nabla \rho|^{q^{\prime}} d x \leq c \int_{0}^{1} \int_{\mathbb{R}^{N-k}}\left|\partial_{t} w\left(y^{2}, x^{\prime \prime}\right)\right|^{q^{\prime}} d x^{\prime} y^{\kappa_{+}+k-1} d y \\
& \leq c \int_{0}^{1} \int_{\mathbb{R}^{N-k}}\left|\partial_{t} w\left(t, x^{\prime \prime}\right)\right|^{q^{\prime}} d x^{\prime} t^{\frac{\kappa_{+}+k}{2}} \frac{d t}{t} \\
& \leq c \int_{0}^{1}\left\|t^{1-\left(\frac{1}{2}-\beta\right)} \frac{d S(t)[\eta]}{d t}\right\|_{L^{q^{\prime}}\left(\mathbb{R}^{N-k}\right)}^{q^{\prime}} \frac{d t}{t} \\
& \approx\|\eta\|_{W^{2(1-\beta), q^{\prime}}\left(\mathbb{R}^{N-k)}\right.}^{q^{\prime}} .
\end{aligned}
$$

This proves (4.28). Further, (4.27) and (4.28) imply (4.15).

We turn to the proof of part (ii). Let $E$ be a closed subset of $B_{R / 2}^{N-k}(0)$ such that $C_{s, q^{\prime}}^{N-k}(E)=0$. Then there exists a sequence $\left\{\eta_{n}\right\}$ in $C_{0}^{\infty}\left(d_{A}\right)$ such that $0 \leq \eta_{n} \leq 1$, $\eta_{n}=1$ in a neighborhood of $E$ (which may depend on $n$ ), supp $\eta_{n} \subset B_{3 R / 4}^{N-k}(0)$ and $\left\|\eta_{n}\right\|_{W^{s, q^{\prime}}} \rightarrow 0$. Then, by (4.28),

$$
\left\|L\left[\eta_{n}\right]\right\|_{L^{q^{\prime}\left(D_{A}^{R}\right)}} \rightarrow 0 .
$$

Furthermore

$$
\|w\|_{L^{q^{\prime}\left((0, R) \times B_{R}^{N-k}(0)\right)}} \leq c\left\|\eta_{n}\right\|_{L^{q^{\prime}\left(B_{R}^{N-k}(0)\right)}}
$$

and consequently

$$
H\left[\eta_{n}\right] \rightarrow 0 \text { in } L^{q^{\prime}}\left(D_{A}^{R}\right) .
$$

(Here we use the fact that $k \geq 2$.) In addition

$$
0 \leq H\left[\eta_{n}\right] \leq 1, \quad H\left[\eta_{n}\right] \leq c\left(R-\left|x^{\prime}\right|\right)
$$

with a constant $c$ independent of $n$. Hence (see (4.20))

$$
\zeta_{n, R}:=\rho_{A} \psi_{R} H\left[\eta_{n}\right]^{q^{\prime}} \leq \rho^{R} \rho_{A} \psi_{R} H\left[\eta_{n}\right]^{q^{\prime}-1} \leq \rho^{R} \rho_{A}^{R} H\left[\eta_{n}\right]^{q^{\prime}-1} .
$$

As $u^{q} \rho^{R} \rho_{A}^{R} \in L^{1}\left(D_{A}^{R}\right)$ we obtain

$$
\lim _{n \rightarrow \infty} \int_{D_{A}} u^{q} \zeta_{n} d x=0
$$

This fact and (4.27) imply that

$$
\int_{d_{A}^{R}} \eta_{n}^{q^{\prime}} d \mu \rightarrow 0
$$

As $\eta_{n}=1$ on a neighborhood of $E$ in $\mathbb{R}^{N-k}$ it follows that $\mu(E)=0$. 
Proposition 4.5. Let $D_{A}$ be a $k$-dihedron, $1 \leq k<N$. Let $k_{+}$be as in (2.11) and let $q_{c}^{*}$ and $q_{c}$ be as in Proposition 2.1 and Proposition 2.2 respectively. Assume that $q_{c} \leq q<q_{c}^{*}$. A measure $\mu \in \mathfrak{M}\left(\partial D_{A}\right)$, with compact support contained in $d_{A}$, is $q$-good relative to $D_{A}$ if and only if $\mu$ vanishes on every Borel set $E \subset d_{A}$ such that $_{s, q^{\prime}}(E)=0$, where $s=2-\frac{k+\kappa_{+}}{q^{\prime}}$.

Remark. We shall use the notation $\mu \prec C_{s, q^{\prime}}$ to say that $\mu$ vanishes on any Borel set $E \subset\left(d_{A}\right)$ such that $C_{s, q^{\prime}}(E)=0$.

In the case $k=N: D_{A}=C_{A}$ (= the cone with vertex 0 and opening $A$ in $\mathbb{R}^{k}$ ) and $q_{c}=q_{c}^{*}$. By [24] (specifically the results quoted in Subsection 2.2) $q_{c}=1-\frac{2}{\kappa_{-}}=\frac{N+\kappa_{+}}{N+\kappa_{+}-2}$ and if $1<q<q_{c}$ then there exist solutions for every measure $\mu=k \delta_{P}, P \in d_{A}$.

In the case $k=1, q_{c}^{*}=\infty, \kappa_{+}=1$ and $q_{c}=\frac{N+1}{N-1}$. Thus $s=2 / q$ and the statement of the theorem is well known (see [21]).

Proof. In view of the last remark, it remains to deal only with $2 \leq k \leq N-1$. We shall identify $d_{A}$ with $\mathbb{R}^{N-k}$.

It is sufficient to prove the result for positive measures because $\mu \prec C_{s, q^{\prime}}$ if and only if $|\mu| \prec C_{s, q^{\prime}}$. In addition, if $|\mu|$ is a $q$-good measure then $\mu$ is a $q$-good measure.

First we show that if $\mu$ is non-negative and $q$-good then $\mu \prec C_{s, q^{\prime}}$. If $E$ is a Borel subset of $\partial \Omega$ then $\mu \chi_{E}$ is $q$-good. If $E$ is compact and $C_{s, q^{\prime}}(E)=0$ then, by Proposition $4.4, E$ is a removable set. This means that the only positive solution of (1.1) in $D_{A}$ such that $\mu(\partial \Omega \backslash E)=0$ is the zero solution. This implies that $\mu \chi_{E}=0$, i.e., $\mu(E)=0$. If $C_{s, q^{\prime}}(E)=0$ but $E$ is not compact then $\mu\left(E^{\prime}\right)=0$ for every compact set $E^{\prime} \subset E$. Therefore, we conclude again that $\mu(E)=0$.

Next, assume that $\mu$ is a positive measure in $\mathfrak{M}\left(\partial D_{A}\right)$ supported in a compact subset of $\mathbb{R}^{N-k}$.

If $\mu \in B^{-s, q}\left(\mathbb{R}^{N-k}\right)$ then, by Theorem $3.1, \mu$ is admissible relative to $D_{A} \cap$ $\Gamma_{k, R}$, for every $R>0$. (As before $\Gamma_{k, R}$ is the cylinder with radius $R$ around the "axis" $\mathbb{R}^{N-k}$.) This implies that $\mu$ is $q$-good relative to $D_{A}$.

If $\mu \prec C_{s, q^{\prime}}$ then, by a theorem of Feyel and de la Pradelle [11] (see also [3]), there exists a sequence $\left\{\mu_{n}\right\} \subset\left(B^{-s, q}\left(\mathbb{R}^{N-k}\right)\right)_{+}$such that $\mu_{n} \uparrow \mu$. As $\mu_{k}$ is $q$ good, it follows that $\mu$ is $q$-good.

Theorem 4.6. Let $P$ be an N-dimensional polyhedron as described in Proposition 4.3. Let $\mu$ be a bounded measure on $\partial P$, (may be a signed measure). Let $k=1, \ldots, N, j=1, \ldots, n_{k}$, and let $L_{k, j}$ and $A_{k, j}$ be defined as at the beginning of this section. Further, put

$$
s(k, j)=2-\frac{k+\left(\kappa_{+}\right)_{k, j}}{q^{\prime}},
$$

where $\left(\kappa_{+}\right)_{k, j}$ is defined as in (2.11) with $A=A_{k, j}$. Then $\mu \in \mathfrak{M}_{q}(\partial P)$, i.e., $\mu$ is a good measure for (1.1) relative to $P$, if and only if, for every pair $(k, j)$ as above and every Borel set $E \subset L_{k, j}$ : 
- If $1 \leq k<N$ then

$$
\begin{aligned}
\left(q_{c}\right)_{k, j} \leq q<\left(q_{c}^{*}\right)_{k, j}, C_{s(k, j), q^{\prime}}^{N-k}(E)=0 & \Longrightarrow \mu(E)=0 \\
q \geq\left(q_{c}^{*}\right)_{k, j} & \Longrightarrow \mu\left(L_{N, j}\right)=0
\end{aligned}
$$

and if $k=N$, i.e., $L$ is a vertex,

$$
q \geq\left(q_{c}\right)_{k, j}=\frac{N+2+\sqrt{(N-2)^{2}+4 \lambda_{A}}}{N-2+\sqrt{(N-2)^{2}+4 \lambda_{A}}} \Longrightarrow \mu(L)=0 .
$$

Here $\left(q_{c}^{*}\right)_{k, j}$ and $\left(q_{c}\right)_{k, j}$ are defined as in (2.32) and (2.36) respectively, with $A=A_{k, j}$.

- If $1<q<\left(q_{c}\right)_{k, j}$ then there is no restriction on $\mu \chi_{L_{k, j}}$.

Proof. This is an immediate consequence of Proposition 4.3 and Proposition 4.5 (see also the Remark following it). In the case $k=N, L_{N, j}$ is a vertex and the condition says merely that for $q \geq\left(q_{c}\right)_{N, j}, \mu$ does not charge the vertex.

\subsection{Removable singular sets, II}

Proposition 4.7. Let $A$ be a Lipschitz domain on $S^{k-1}, 2 \leq k \leq N-1$, and let $D_{A}$ be the $k$-dihedron with opening $A$. Let $u$ be a positive solution of (1.1) in $D_{A}^{R}$, for some $R>0$. Suppose that $F=\mathcal{S}(u) \subset d_{A}^{R}$ and let $Q$ be an open neighborhood of $F$ such that $\bar{Q} \subset d_{A}^{R}$. (Recall that $d_{A}^{R}=d_{A} \cap B_{R}^{N-k}(0)$ is an open subset of $d_{A}$.) Let $\mu$ be the trace of $u$ on $\mathcal{R}(u)$.

Let $\eta \in W_{0}^{s, q^{\prime}}\left(d_{A}^{R}\right)$ such that

$$
0 \leq \eta \leq 1, \quad \eta=0 \text { on } Q .
$$

Employing the notation in the proof of Proposition 4.4, put

$$
\zeta:=\rho_{A} \psi_{R} H_{R}[\eta]^{q^{\prime}}
$$

Then

$$
\int_{D_{A}^{R}} u^{q} \zeta d x \leq c\left(1+\|\eta\|_{W^{s, q^{\prime}\left(d_{A}\right)}}\right)^{q^{\prime}}+\mu\left(d_{A}^{R} \backslash Q\right)^{q},
$$

$c$ independent of $u$ and $\eta$.

Proof. First we prove (4.34) for $\eta \in C_{0}^{\infty}\left(d_{A}^{R}\right)$. Let $\sigma_{0}$ be a point in $A$ and let $\left\{A_{n}\right\}$ be a Lipschitz exhaustion of $A$. If $0<\epsilon<\operatorname{dist}\left(\partial A, \partial A_{n}\right)=\bar{\epsilon}_{n}$ then

$$
\epsilon \sigma_{0}+C_{A_{n}} \subset C_{A} .
$$

Denote

$$
D_{A}^{R^{\prime}, R^{\prime \prime}}=D_{A} \cap\left[\left|x^{\prime}\right|<R^{\prime}\right] \cap\left[\left|x^{\prime \prime}\right|<R^{\prime \prime}\right] .
$$


Pick a sequence $\left\{\epsilon_{n}\right\}$ decreasing to zero such that $0<\epsilon_{n}<\min \left(\bar{\epsilon}_{n} / 2^{n}, R / 8\right)$. Let $u_{n}$ be the function given by

$$
u_{n}\left(x^{\prime} x^{\prime \prime}\right)=u\left(x^{\prime}+\epsilon_{n} \sigma_{0}, x^{\prime \prime}\right) \quad \forall x \in D_{A_{n}}^{R_{n}, R}, \quad R_{n}=R-\epsilon_{n} .
$$

Then $u_{n}$ is a solution of (1.1) in $D_{A_{n}}^{R_{n}, R}$ belonging to $C^{2}\left(\bar{D}_{A_{n}}^{R_{n}, R}\right)$ and we denote its boundary trace by $h_{n}$. Let

$$
\zeta_{n}:=\rho_{A_{n}} \psi_{R} H_{R}[\eta]^{q^{\prime}}
$$

with $\psi_{R}$ and $H_{R}[\eta]$ as in the proof of Proposition 4.4. By Proposition 4.2

$$
-\int_{D_{A_{n}}^{R_{n}, R}} \mathbb{P}\left[h_{n}\right] \Delta \zeta_{n} d x=\int_{B_{R}^{N-k}(0)} \eta^{q^{\prime}} h_{n} d \omega_{n}
$$

where $\omega_{n}$ is the harmonic measure on $d_{A_{n}}^{R}$ relative to $D_{A_{n}}^{R_{n}, R}$. (Note that $d_{A_{n}}^{R}=d_{A}^{R}$ and we may identify it with $B_{R}^{N-k}(0)$.) Hence

$$
\int_{D_{A_{n}}^{R_{n}, R}}\left(-u_{n} \Delta \zeta_{n}+u_{n}^{q} \zeta_{n}\right) d x=-\int_{B_{R}^{N-k}(0)} \eta^{q^{\prime}} h_{n} d \omega_{n}
$$

Further,

$$
\int_{B_{R}^{N-k}(0)} \eta^{q^{\prime}} h_{n} d \omega_{n} \rightarrow \int_{B_{R}^{N-k}(0)} \eta^{q^{\prime}} d \mu \leq \mu\left(d_{A}^{R} \backslash Q\right),
$$

because $\eta=0$ in $Q$. By (4.24), (4.28) we obtain,

$$
\begin{aligned}
& \left|\int_{D_{A_{n}}^{R_{n}, R}} u_{n} \Delta \zeta_{n} d x\right| \\
\leq & c\left(\int_{D_{A_{n}}^{R_{n}, R}} u_{n}^{q} \zeta_{n} d x\right)^{\frac{1}{q}}\left(\left(\int_{D_{A_{n}}^{R_{n}, R}} \zeta_{n} d x\right)^{\frac{1}{q^{\prime}}}+\|\eta\|_{W^{s, q^{\prime}}\left(B_{R}^{N-k}(0)\right)}\right) .
\end{aligned}
$$

From the definition of $\zeta_{n}$ it follows that

$$
\int_{D_{A_{n}}^{R_{n}, R}} \zeta_{n} d x \leq \int_{D_{A_{n}}^{R_{n}, R}} \rho_{n} d x \rightarrow \int_{D_{A}^{R}} \rho d x
$$

where $\rho$ (respectively $\rho_{n}$ ) is the first eigenfunction of $-\Delta$ in $D_{A}^{R}$ (respectively $D_{A_{n}}^{R_{n}, R}$ ) normalized by 1 at some $x_{0} \in D_{A_{1}}^{R_{1}, R}$. Therefore, by (4.36),

$$
\int_{D_{A_{n}}^{R_{n}, R}} u_{n}^{q} \zeta_{n} d x \leq c\left(\int_{D_{A_{n}}^{R_{n}, R}} u_{n}^{q} \zeta_{n} d x\right)^{\frac{1}{q}}\left(1+\|\eta\|_{W^{s, q^{\prime}}\left(B_{R}^{N-k}(0)\right)}\right)+\mu\left(d_{A}^{R} \backslash Q\right) .
$$


This implies

$$
\int_{D_{A_{n}}^{R_{n}, R}} u_{n}^{q} \zeta_{n} d x \leq c\left(1+\|\eta\|_{W^{s, q^{\prime}}\left(B_{R}^{N-k}(0)\right)}\right)^{q^{\prime}}+\mu\left(d_{A}^{R} \backslash Q\right)^{q} .
$$

To verify this fact, put

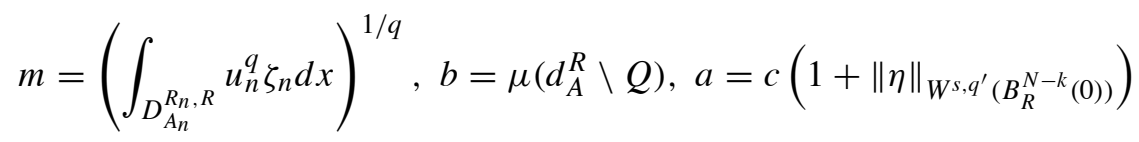

so that (4.38) becomes

$$
m^{q}-a m-b \leq 0 .
$$

If $b \leq m$ then

$$
m^{q-1}-a-1 \leq 0 .
$$

Therefore,

$$
m \leq(a+1)^{\frac{1}{q-1}}+b
$$

which implies (4.38). Finally, by the lemma of Fatou we obtain (4.34) for $\eta \in C_{0}^{\infty}$. By continuity we obtain the inequality for any $\eta \in W_{0}^{s, q^{\prime}}$ satisfying (4.32).

Theorem 4.8. Let $A$ be a Lipschitzdomain on $S^{k-1}, 2 \leq k \leq N-1$, and let $D_{A}$ be the $k$-dihedron with opening $A$. Let $E$ be a compact subset of $d_{A}^{R}$ and let $u$ be a non-negative solution of (1.1) in $D_{A}^{R}$ (for some $R>0$ ) such thatu vanishes on $\partial D_{A}^{R} \backslash E$. Then

$$
C_{s, q^{\prime}}^{N-k}(E)=0, \quad s=2-\frac{\kappa_{+}+k}{q^{\prime}} \Longrightarrow u=0,
$$

where $C_{s, q^{\prime}}^{N-k}$ denotes the Bessel capacity with the indicated indices in $\mathbb{R}^{N-k}$.

Proof. By Proposition 4.4, (4.39) holds under the additional assumption

$$
\int_{D_{A}^{R}} u^{q} \rho_{R} \rho_{A}^{R} d x<\infty
$$

Indeed, by [24, Proposition 4.1], (4.40) implies that the solution $u$ possesses a boundary trace $\mu$ on $\partial D_{A}^{R}$. By assumption, $\mu\left(\partial D_{A}^{R} \backslash E\right)=0$. Therefore, by Proposition 4.5, the fact that $C_{s, q^{\prime}}^{N-k}(E)=0$ implies that $\mu(E)=0$. Thus $\mu=0$ and hence $u=0$.

We show that, under the conditions of the theorem, if $C_{s, q^{\prime}}^{N-k}(E)=0$ then (4.40) holds. 
By Proposition 4.7, for every $\eta \in W_{0}^{s, q^{\prime}}\left(d_{A}^{R}\right)$ such that $0 \leq \eta \leq 1$ and $\eta=$ 0 in a neighborhood of $E$,

$$
\int_{D_{A}^{R}} u^{q} \zeta d x \leq c\left(1+\|\eta\|_{W^{s, q^{\prime}}\left(B_{R}^{N-k}(0)\right)}\right)^{q^{\prime}},
$$

for $\zeta$ as in (4.33). (Here we use the assumption that $u=0$ on $\partial D_{A}^{R} \backslash E$.)

Let $a>0$ be sufficiently small so that $E \subset B_{(1-4 a) R}^{N-k}(0)$. Pick a sequence $\left\{\phi_{n}\right\}$ in $C_{0}^{\infty}\left(\mathbb{R}^{N-k}\right)$ such that, for each $n$, there exists a neighborhood $Q_{n}$ of $E$, $\bar{Q}_{n} \subset B_{(1-3 a) R}^{N-k}(0)$ and

$$
\begin{aligned}
& 0 \leq \phi_{n} \leq 1 \text { everywhere, } \phi_{n}=1 \text { in } Q_{n} \\
& \tilde{\phi}_{n}:=\phi_{n} \chi_{\left[\left|x^{\prime \prime}\right|<(1-2 a) R\right]} \in C_{0}^{\infty}\left(\mathbb{R}^{N-k}\right) \\
& \left\|\tilde{\phi}_{n}\right\|_{W^{s, q^{\prime}}\left(\mathbb{R}^{N-k}\right)} \rightarrow 0 \text { as } n \rightarrow \infty \\
& \eta_{n}:=\left(1-\phi_{n}\right)\left\lfloor_{\left[\left|x^{\prime \prime}\right|<R\right]} \in C_{0}^{\infty}\left(d_{A}^{R}\right)\right. \\
& \eta_{n}=0 \text { in }\left[(1-a) R<\left|x^{\prime \prime}\right|<R\right] .
\end{aligned}
$$

Such a sequence exists because $C_{s, q^{\prime}}^{N-k}(E)=0$. Applying (4.41) to $\eta_{n}$ we obtain,

$$
\sup \int_{D_{A}^{R}} u^{q} \zeta_{n} d x \leq c<\infty
$$

where $\zeta_{n}=\rho_{A} \psi_{R} H_{R}^{q^{\prime}}\left[\eta_{n}\right]$ (see (4.33)). By taking a subsequence we may assume that $\left\{\eta_{n}\right\}$ converges (say to $\eta$ ) in $L^{q^{\prime}}\left(B_{R}^{N-k}(0)\right)$ and consequently $H\left[\eta_{n}\right] \rightarrow H[\eta]$ in the sense that

$$
H_{R}\left[\eta_{n}\right]\left(x^{\prime}, \cdot\right)=w_{n, R}\left(y^{2}, \cdot\right) \rightarrow w_{R}\left(y^{2}, \cdot\right)=H_{R}[\eta]\left(x^{\prime}, \cdot\right) \text { in } L^{q^{\prime}}
$$

uniformly with respect to $y=\left|x^{\prime}\right|$. It follows that

$$
\int_{D_{A}^{R}} u^{q} \zeta d x<\infty, \quad \zeta=\rho_{A} \psi_{R} H_{R}^{q^{\prime}}[\eta] .
$$

As $\tilde{\phi}_{n} \rightarrow 0$ in $W^{s, q^{\prime}}\left(\mathbb{R}^{N-k}\right)$ it follows that $\phi_{n} \rightarrow 0$ and hence $\eta_{n} \rightarrow 1$ a.e. in $B_{(1-2 a) R}^{N-k}(0)$. Thus $\eta=1$ in this ball, $\eta=0$ in $\left[(1-a) R<\left|x^{\prime \prime}\right|<R\right]$ and $0 \leq \eta \leq 1$ everywhere.

Consequently, given $\delta>0$, there exists an $N$-dimensional neighborhood $O$ of $d_{A} \cap B_{(1-2 a) R}^{N-k}(0)$ such that

$$
1-\delta<H_{R}[\eta]<1 \text { and } 1-\delta<\psi_{R} / \rho_{A}^{R}<1 \text { in } O .
$$


Therefore (4.44) implies that

$$
\int_{D_{A}^{(1-3 a) R}} u^{q} \rho^{R} \rho_{A}^{R} d x \leq c<\infty .
$$

Recall that the trace of $u$ on $\partial D_{A}^{R} \backslash d_{A}^{(1-4 a) R}$ is zero. Therefore $u$ is bounded in $D_{A}^{R} \backslash D_{A}^{(1-3 a) R}$. This fact and (4.45) imply (4.40).

Definition 4.9. Let $\Omega$ be a bounded Lipschitz domain. Denote by $\rho$ the first eigenfunction of $-\Delta$ in $\Omega$ normalized by $\rho\left(x_{0}\right)=1$ for a fixed point $x_{0} \in \Omega$.

For every compact set $K \subset \partial \Omega$ we define

$$
M_{\rho, q}(K)=\left\{\mu \in \mathfrak{M}(\partial \Omega): \mu \geq 0, \mu(\partial \Omega \backslash K)=0, \mathbb{K}[\mu] \in L_{\rho}^{q}(\Omega)\right\}
$$

and

$$
\tilde{C}_{\rho, q^{\prime}}(K)=\sup \left\{\mu(K)^{q}: \mu \in M_{\rho, q}(K), \int_{\Omega} \mathbb{K}[\mu]^{q} \rho d x=1\right\} .
$$

Finally we denote by $C_{\rho, q^{\prime}}$ the outer measure generated by the above functional.

The following statement is verified by standard arguments:

Lemma 4.10. For every compact $K \subset \partial \Omega, C_{\rho, q^{\prime}}(K)=\tilde{C}_{\rho, q^{\prime}}(K)$. Thus $C_{\rho, q^{\prime}}$ is a capacity and,

$$
C_{\rho, q^{\prime}}(K)=0 \Longleftrightarrow M_{\rho, q}(K)=\{0\} \text {. }
$$

Theorem 4.11. Let $\Omega$ be a bounded polyhedron in $\mathbb{R}^{N}$. A compact set $K \subset \partial \Omega$ is removable if and only if

$$
C_{s(k, j), q^{\prime}}\left(K \cap L_{k, j}\right)=0,
$$

for $k=1, \cdot, N j=1, \ldots, n_{k}$, where $s(k, j)$ is defined as in (4.29). This condition is equivalent to

$$
C_{\rho, q^{\prime}}(K)=0 .
$$

A measure $\mu \in \mathfrak{M}(\partial \Omega)$ is q-good if and only if it does not charge sets with $C_{\rho, q^{\prime-}}$ capacity zero.

Proof. The first assertion is an immediate consequence of Proposition 4.3 and Theorem 4.8. The second assertion follows from the fact that

$$
C_{\rho, q^{\prime}}\left(K \cap L_{k, j}\right)=C_{s(k, j), q^{\prime}}\left(K \cap L_{k, j}\right) .
$$

The third assertion follows from Theorem 4.6 and the previous statement. 


\section{References}

[1] D. R. Adams and L. I. Hedberg, "Function Spaces and Potential Theory", Grundl. Math Wiss., Vol. 314, Springer-Verlag, Berlin, 1966.

[2] A. AnCona and M. MARCUS, Positive solutions of a class of semilinear equations with absorption and schrödinger equations, J. Math. Pures Appl. 104 (2015), 587-618.

[3] P. BARAS and M. PIERRE, Singularités éliminables pour des équations semi-lineaires, Ann. Inst. Fourier (Grenoble) 34 (1984), 185-206.

[4] Bui HuY Qui, Harmonic functions, Riesz potentials, and the Lipschitz spaces of Herz, Hiroshima Math. J. 9 (1979), 245-295.

[5] A.P. CALDERON, Lebesgue spaces of differentiable functions and distributions, In: "Partial Differential Equations", Proc. Sympos. Pure Math., Vol. 4, Amer. Math. Soc., Providence, RI, 1961,33-49.

[6] E. B. Dynkin, "Diffusions, Superdiffusions and Partial Differential Equations", Amer. Math. Soc. Colloquium Publications, Vol. 50, Providence, RI, 2002.

[7] E. B. DYNKIn, "Superdiffusions and Positive Solutions of Nonlinear Partial Differential Equations, University Lecture Series, Vol. 34, Amer. Math. Soc., Providence, RI, 2004.

[8] E. B. DYNKIN and S. E. KuZNETSOV, Superdiffusions and removable singularities for quasilinear partial differential equations, Comm. Pure Appl. Math. 49 (1996), 125-176.

[9] E. B. DYNKIN and S. E. KUZNETSOV, Fine topology and fine trace on the boundary associated with a class of quasilinear differential equations, Comm. Pure Appl. Math. 51 (1998), 897-936.

[10] J. FABBRI and L. VÉRON, Singular boundary value problems for nonlinear elliptic equations in non smooth domains, Adv. Differential Equations 1 (1996), 1075-1098.

[11] D. FeYEL and A. DE LA PRADELle, Topologies fines et compactifications associŐes Ĺ certains espaces de Dirichlet, Ann. Inst. Fourier (Grenoble) 27 (1977), 121-146.

[12] K. GKIKAS and L. VERON, Boundary singularities of solutions of semilinear elliptic equations with critical Hardy potentials, Nonlinear Anal. 121 (2015), 469-540.

[13] N. Gilbarg and N. S. TRUdinger, "Partial Differential Equations of Second Order", 2nd ed., Springer-Verlag, Berlin/New-York, 1983.

[14] C. KENIG and J. PIPHER, The h-path distribution of conditioned Brownian motion for nonsmooth domains, Probab. Theory Related Fields 82 (1989), 615-623.

[15] J. B. Keller, On solutions of $\Delta u=f(u)$, Comm. Pure Appl. Math. 10 (1957), 503-510.

[16] J. F. LE GALL, The Brownian snake and solutions of $\Delta u=u^{2}$ in a domain, Probab. Theory Related Fields 102 (1995), 393-432.

[17] J. F. LE GALL, "Spatial Branching Processes, Random Snakes and Partial Differential Equations", Lectures in Mathematics ETH Zürich, Birkhäuser Verlag, Basel, 1999.

[18] M. MARCUS, Complete classication of the positive solutions of $-\Delta u+u^{q}=0$, J. Anal. Math. 117 (2012), 187-220.

[19] M. MARCUS and L. VÉRON, The boundary trace of positive solutions of semilinear elliptic equations: the subcritical case, Arch. Ration. Mech. An. 144 (1998), 201-231.

[20] M. MARCUS and L. VÉRON, The boundary trace of positive solutions of semilinear elliptic equations: the supercritical case, J. Math. Pures Appl. 77 (1998), 481-521.

[21] M. MARCUS and L. VÉRON, Removable singularities and boundary traces, J. Math. Pures Appl. 80 (2001), 879-900.

[22] M. MARCUS and L. VÉRON The boundary trace and generalized boundary value problem for semilinear elliptic equations with coercive absorption, Comm. Pure Appl. Math. 56 (2003), 689-731.

[23] M. MARCUS and L. VÉRON, The precise boundary trace of positive solutions of the equation $\Delta u=u^{q}$ in the supercritical case, In: "Perspectives in Nonlinear Partial Differential Equations", Berestycki Henri et al. (eds.), Based on the Conference celebration of Häim Bretis' 60th birthday, June 21-25, 2004, Amer. Mathematical Society (ANS), Contemporay Mathemathics vol. 446, Providence, RI, 2007, 345-383. 
[24] M. MARCUS and L. VÉRON, Boundary trace of positive solutions of semilinear elliptic equations in Lipschitz domains: the subcritrical case, Annali Sc. Norm. Super. Pisa, Classe di Scienze, Ser. V X (2011), 913-984.

[25] B. MsElatI, "Classification and Probabilistic Representation of the Positive Solutions of a Semilinear Elliptic Equation”, Mem. Amer. Math. Soc., Vol. 168, 2004.

[26] R. Osserman, On the inequality $\Delta u \geq f(u)$, Pacific J. Math. 7 (1957), 1641-1647.

[27] E. STEIN, "Singular Integral and Differentiability Properties of Functions", Princeton Univ. Press, 1970.

[28] H. TRIEbel, "Interpolation Theory, Function Spaces, Differential Operators", NorthHolland Pub. Co., 1978.

Department of Mathematics

Technion - Israel Institute of Technology

Haifa, 32000, Israel

marcusm@math.technion.ac.il

Laboratoire de Mathématiques

Faculté des Sciences

Parc de Grandmont

37200 Tours, France

veronl@lmpt.univ-tours.fr 